\title{
Variable Lorentz estimate for nonlinear elliptic equations with partially regular nonlinearities
}

\author{
Shuang Liang* Shenzhou Zheng ${ }^{\dagger}$
}

January 12, 2018

\begin{abstract}
We prove global Calderón-Zygmund type estimate in Lorentz spaces for variable power of the gradients to weak solution of nonlinear elliptic equations in a non-smooth domain. We mainly assume that the nonlinearities are merely measurable in one of the spatial variables and have sufficiently small BMO semi-norm in the other variables, the boundary of domain belongs to Reifenberg flatness, and the variable exponents $p(x)$ satisfy log-Hölder continuity.
\end{abstract}

MR(2010) Subject Classification: 35D30; 35K10

Key words: nonlinear elliptic equations; Lorentz estimates for variable power of gradients; partially BMO; Reifenberg flatness

\section{Introduction}

Throughout this paper, let $\Omega$ be a bounded domain $\mathbb{R}^{n}(n \geq 2)$ with a rough boundary specified later. Suppose that $\mathbf{F}=\left(f^{1}, f^{2}, \cdots, f^{n}\right)$ is a given vector-valued measurable function, and $\mathbf{a}=\mathbf{a}(\xi, x): \mathbb{R}^{n} \times \Omega \rightarrow \mathbb{R}^{n}$ is a Carathéodory vector valued function which is measurable in $x \in \Omega$ for each $\xi \in \mathbb{R}^{n}$ and Lipschitz continuous in $\xi \in \mathbb{R}^{n}$ for each $x \in \Omega$. The aim of this article is to study a global Lorentz estimate for variable power of the gradients to weak solution of the Dirichlet problem for nonlinear elliptic equations:

$$
\begin{cases}\operatorname{div} \mathbf{a}(D u, x)=\operatorname{div} \mathbf{F}, & \text { in } \Omega, \\ u=0 & \text { on } \partial \Omega\end{cases}
$$

under very weak assumptions that the nonlinearities $\mathbf{a}(\xi, x)$ are merely small partially BMO (Bounded Mean Oscillation) semi-norm in the spatial variables and $\partial \Omega$ is Reifenberg flat. The weak solution of (1.1) is understood in the usual sense: for $u \in W_{0}^{1,2}(\Omega)$ it holds

$$
\int_{\Omega}\langle\mathbf{a}(D u, x), D \varphi\rangle d x=\int_{\Omega}\langle\mathbf{F}, D \varphi\rangle d x, \quad \forall \varphi \in W_{0}^{1,2}(\Omega) .
$$

To ensure solvability in $L^{2}(\Omega)$ of (1.1), we need to impose a structural assumption with ellipticity and growth: there exist two constants $0<v \leq \Lambda<\infty$ such that

$$
\left\{\begin{array}{l}
\left\langle D_{\xi} \mathbf{a}(\xi, x) \eta \cdot \eta\right\rangle \geq v|\eta|^{2}, \\
|\mathbf{a}(\xi, x)|+|\xi|\left|D_{\xi} \mathbf{a}(\xi, x)\right| \leq \Lambda|\xi|
\end{array}\right.
$$

\footnotetext{
*Department of Mathematics, Beijing Jiaotong University, Beijing 100044, China. email:shuangliang@ bjtu.edu.cn.

†Corresponding author: 1 Department of Mathematics, Beijing Jiaotong University, Beijing 100044, China; email:shzhzheng@bjtu.edu.cn; Tel:8610-51682054-118. ${ }^{2}$ BCAM-Basque Center for Applied Mathematics, Alameda de Mazarredo 14, 48009 Bilbao, Spain.
} 
for a. e. $x \in \Omega$ and $\xi, \eta \in \mathbb{R}^{n}$, where $D_{\xi}$ denotes the differentiation in $\xi \in \mathbb{R}^{n}$, and $\langle\cdot, \cdot\rangle$ is the standard inner product in $\mathbb{R}^{n}$. By (1.3) it is clear to check that

$$
\left\{\begin{array}{l}
\mathbf{a}(0, x)=0, \\
v|\xi-\eta|^{2} \leq\langle\mathbf{a}(\xi, x)-\mathbf{a}(\eta, x), \xi-\eta\rangle .
\end{array}\right.
$$

Therefore, by the usual Minty-Browder argument there exists a unique weak solution $u \in W_{0}^{1,2}(\Omega)$ of $(1.1)$ with the following $L^{2}$ estimate

$$
\|D u\|_{L^{2}(\Omega)} \leq c\|\mathbf{F}\|_{L^{2}(\Omega)},
$$

where $c$ is a constant independent of $u, \mathbf{F}$ and $\Omega$.

The Calderón-Zygmund theory concerned partial differential equations with partially regular coefficient assumptions has been getting largely attention. For the case of linear PDEs, this was first introduced by Kim and Krylov in [19], and later employed by Dong and Kim in [14, 16, 17] and by Byun and Wang in [6] in the study of Calderón-Zygmund theory to divergence and nondivergence linear elliptic and parabolic equations/systems with partially VMO or small partially BMO coefficients. It has actually proved to be a sort of minimal regular requirement imposed on the leading coefficients for elliptic and parabolic operators to ensure a satisfactory Calderón-Zygmund theory for all $p>1$. Indeed, this was verified due to a famous counterexample by Ural'tseva [29], who constructed an example of an equation in $\mathbb{R}^{d}(d \geq 3)$ with the coefficients depending only on the first two coordinates so that we reached that there is no unique solvability in Sobolev spaces $W^{1, p}$ for any $p>1$. It is worth noting that Byun-Wang [6] and Byun-Palagachev [10] considered linear elliptic equations with partially BMO coefficients and obtained the $L^{p}$-estimate, weighted $L^{p}$-estimate, respectively. We are now interested in nonlinear elliptic equations with partially regular nonlinearities in the spatial variables since those are related to nonlinear problems in medium composition materials. We would particularly like to point out that the study of this article was inspired by two recent progresses from Byun et al's papers. Byun, Ok and Wang [9] obtained a global $L^{p(x)}$ estimate to the Dirichlet problem of divergence linear elliptic system in Reifenberg domain with partially BMO coefficients and log-Hölder continuity $p(x)$, who showed that

$$
\mathbf{F} \in L^{p(x)}\left(\Omega, \mathbb{R}^{n}\right) \Rightarrow D u \in L^{p(x)}\left(\Omega, \mathbb{R}^{n}\right), \quad p(x) \geq 2 .
$$

On the other hand, Byun and Kim [11] also established global $L^{p}$ theory to divergence nonlinear elliptic equations (1.1) with measurable nonlinearities, which means that

$$
\mathbf{F} \in L^{p}(\Omega) \Rightarrow D u \in L^{p}(\Omega), \quad 2 \leq p<\infty
$$

for weak solution $u \in W_{0}^{1,2}(\Omega)$ of the Dirichlet problems (1.1). Therefore, a refined natural outgrowth of the above-mentioned two papers leads to our consideration in the framework of variable Lorentz spaces.

As we know, Lorentz spaces are a two-parameter scale of the Lebesgue spaces by refining Lebesgue spaces in the fashion of second index. Recently there were a large of literatures on the topic concerning Lorentz regularity of PDEs. For instance, Baroni [3] considered the gradient estimate in the scale of Lorentz spaces to parabolic system of $p$-Laplacian type with VMO "coefficients", and he made use of the large-Minequality principle to obtain that

$$
|\mathbf{F}| \in L(\gamma, q) \text { locally in } \Omega_{T} \Rightarrow|D u| \in L(\gamma, q) \text { locally in } \Omega_{T}
$$

with $\gamma>p$ and $q \in(0, \infty]$. Similarly, he also showed that there were gradient Lorentz estimates to degenerate elliptic system and obstacle parabolic problems in [4], respectively. Later, Mengesha-Phuc [21] and Zhang-Zhou [32] derived gradient weighted Lorentz estimates for quasilinear equations of $p$-Laplacian type 
and $p(x)$-Laplacian type by a rather different geometrical approach from $[6,10]$, respectively. Very recently, Tian-Zheng [28] showed global weighted Lorentz estimate to linear elliptic equations with lower order items with partially BMO coefficients and Reifenberg flat domain. Zhang-Zheng [30, 31] also studied with Hessian Lorentz estimates for fully nonlinear parabolic and elliptic equations with small BMO nonlinearities, and Hessian weighted Lorentz estimates of strong solution for nondivergence linear elliptic equations with partially BMO coefficients. We notice that for these papers concerning nonlinear problems mentioned above, an important regular assumption on the "nonlinearity coefficients" is an VMO or small BMO in all $x$ beyond the settings of linear PDEs. To this end, let us start with related basic notations which will be useful in this paper. The Lorentz space $L(t, q)(U)$ for open subset $U \subset \mathbb{R}^{n}$ with parameters $1 \leq t<\infty, 0<q<\infty$, is the set of measurable functions $g: U \rightarrow \mathbb{R}$ by requiring

$$
\|g\|_{L(t, q)(U)}^{q}:=q \int_{0}^{\infty}\left(\mu^{t}|\{\xi \in U:|g(\xi)|>\mu\}|\right)^{\frac{q}{t}} \frac{d \mu}{\mu}<\infty ;
$$

while the Lorentz space $L(t, \infty)$ for $1 \leq t<\infty$ and $q=\infty$ is defined by the Marcinkiewicz space $\mathcal{M}^{t}(U)$ as usual, which is the set of measurable functions $g$ with

$$
\|g\|_{L(t, \infty)}=\|g\|_{\mathcal{M}^{t}(U)}:=\sup _{\mu>0}\left(\mu^{t}|\{\xi \in U:|g(\xi)|>\mu\}|\right)^{\frac{1}{t}}<\infty .
$$

The local variant of such spaces is defined in the usual way. We remark that if $t=q$, then the Lorentz space $L(t, t)(U)$ is nothing but a classical Lebesgue space. Indeed, by Fubini's theorem it yields

$$
\|g\|_{L^{t}(U)}^{t}=t \int_{0}^{\infty} \mu^{t}|\{\xi \in U:|g(\xi)|>\mu\}| \frac{d \mu}{\mu}=\|g\|_{L(t, t)(U)}^{t},
$$

which implies $L^{t}(U)=L(t, t)(U)$, also see $[3,4,5]$.

Note that the main point in this paper is that the exponent $p(x)$ is a variable function. Sharapudinov [26] was the first person to consider a regular hypothesis of variable exponent $p(x)$ satisfying log-Hölder continuity, which ensures the boundedness of Hardy-Littlewood maximal operator in the framework of generalized Lebesgue spaces and basic operations available in the theory of harmonic analysis and PDEs. For this, we recall that $p(x)$ is log-Hölder continuous, denote it by $p(x) \in L H(\Omega)$, if there exist constants $c_{0}$ and $\delta>0$ such that for all $x, y \in \Omega$ with $|x-y|<\delta$, one has

$$
|p(x)-p(y)| \leq \frac{c_{0}}{-\log (|x-y|)} .
$$

Also, the log-Hölder continuity of variable exponent is unavoidable while one treats regularity for elliptic and parabolic problems in the variable exponent Lebesgue spaces. In what follows, we assume that $p(x)$ : $\Omega \rightarrow \mathbb{R}$ is a log-Hölder continuous function; moreover, there exist positive constants $\gamma_{1}$ and $\gamma_{2}$ such that

$$
2<\gamma_{1} \leq p(x) \leq \gamma_{2}<\infty \text { and }|p(x)-p(y)| \leq \omega(|x-y|), \quad \forall x, y \in \Omega,
$$

where $\omega:[0, \infty) \rightarrow[0, \infty)$ is a modulus of continuity of $p(x)$. Without loss of generality, we suppose that $\omega$ is a nondecreasing continuous function with $\omega(0)=0$, and $\limsup _{r \rightarrow 0} \omega(r) \log \left(\frac{1}{r}\right)<\infty$. With the above assumptions in hand, it is also clear that $p(x) \in L H(\Omega)$ and there exists a positive number $A$ such that

$$
\omega(r) \log \left(\frac{1}{r}\right) \leq A \Longleftrightarrow r^{-\omega(r)} \leq e^{A} \quad \text { for any } r \in(0,1) .
$$

Before stating main results, let us recall some basic concepts and facts. We denote a type point by $x=\left(x^{1}, x^{\prime}\right)=\left(x^{1}, x^{2}, \cdots, x^{n}\right) \in \mathbb{R}^{n}$. Let $B_{r}=\left\{x \in \mathbb{R}^{n}:|x|<r\right\}, B_{r}^{+}=B_{r} \cap\left\{x \in \mathbb{R}^{n}: x^{1}>0\right\}$ and 
$B_{r}^{\prime}=\left\{x^{\prime} \in \mathbb{R}^{n-1}:\left|x^{\prime}\right|<r\right\}$ with $B_{r}(y)=B_{r}+y, B_{r}^{+}(y)=B_{r}^{+}+y$ and $B_{r}^{\prime}(y)=B_{r}^{\prime}+y^{\prime}$. Denote typical cylinders $Q_{r}=(-r, r) \times B_{r}^{\prime}, Q_{r}^{+}=Q_{r} \cap\left\{x \in \mathbb{R}^{n}: x^{1}>0\right\}$ with $Q_{r}(y)=Q_{r}+y, Q_{r}^{+}(y)=Q_{r}^{+}+y$; and some typical boundaries $\Omega_{r}(y)=Q_{r}(y) \cap \Omega, \partial_{\omega} \Omega_{r}(y)=Q_{r}(y) \cap \partial \Omega, T_{r}=Q_{r} \cap\left\{x^{1}=0\right\}$. We denote an average of $f$ on $Q_{r}$ for $r>0$ by

$$
f_{Q_{r}} f(x) d x=\frac{1}{\left|Q_{r}\right|} \int_{Q_{r}} f(x) d x
$$

where $\left|Q_{r}\right|$ is $n$-dimensional Lebesgue measure of $Q_{r}$, and an $(n-1)$-dimensional average with respect to $x^{\prime}$ by

$$
\bar{f}_{B_{r}^{\prime}}\left(x_{1}\right)=f_{B_{r}^{\prime}} f\left(x_{1}, x^{\prime}\right) d x^{\prime}=\frac{1}{\left|B_{r}^{\prime}\right|} \int_{B_{r}^{\prime}} f\left(x_{1}, x^{\prime}\right) d x^{\prime}
$$

with $\left|B_{r}^{\prime}\right|$ being the $(n-1)$-dimensional Lebesgue measure of $B_{r}^{\prime}$.

To impose a partially regular assumption on the nonlinearities $\mathbf{a}(\xi, x)=\mathbf{a}\left(\xi, x^{1}, x^{\prime}\right)$, we consider a function

$$
\theta\left(\mathbf{a}, Q_{r}(y)\right)=\sup _{\xi \in \mathbb{R}^{n} \backslash 0} \frac{\left|\mathbf{a}\left(\xi, x^{1}, x^{\prime}\right)-\overline{\mathbf{a}}_{Q_{r}^{\prime}\left(y^{\prime}\right)}\left(\xi, x^{1}\right)\right|}{|\xi|^{2}}
$$

with

$$
\overline{\mathbf{a}}_{Q_{r}^{\prime}\left(y^{\prime}\right)}\left(\xi, x^{1}\right)=f_{Q_{r}^{\prime}\left(y^{\prime}\right)} \mathbf{a}\left(\xi, x^{1}, z^{\prime}\right) d z^{\prime} .
$$

Assumption 1.1 Let $0<\delta<1 / 8$ and $R>0$. We say that $(\boldsymbol{a}(\xi, x), \Omega)$ is $(\delta, R)$-vanishing of codimension 1 if for every point $x_{0} \in \Omega$, there exists a constant $R>0$ such that for any $0<r \leq R$ with

$$
\operatorname{dist}\left(x_{0}, \partial \Omega\right)=\min _{z \in \partial \Omega} \operatorname{dist}\left(x_{0}, z\right)>\sqrt{2} r,
$$

one has that there exists a coordinate system depending only on $x_{0}$ and $r$, whose variables are still denoted by $x$, such that in the new coordinate system with $x_{0}$ as the origin and

$$
f_{Q_{r}}\left|\theta\left(\boldsymbol{a}, Q_{r}\right)(x)\right|^{2} d x \leq \delta^{2}
$$

while, for $x_{0} \in \bar{\Omega}$ with

$$
\operatorname{dist}\left(x_{0}, \partial \Omega\right)=\min _{z \in \partial \Omega} \operatorname{dist}\left(x_{0}, z\right)=\operatorname{dist}\left(x_{0}, z_{0}\right) \leq \sqrt{2} r,
$$

where $z_{0} \in \partial \Omega$, one has that there exists a coordinate system depending on $x_{0}$ and $0<r<R_{0}$ so that in the new coordinate system $z_{0}$ as the origin with

$$
Q_{3 r} \cap\left\{x_{1} \geq 3 \delta r\right\} \subset Q_{3 r} \cap \Omega \subset Q_{3 r} \cap\left\{x_{1} \geq-3 \delta r\right\}
$$

and

$$
f_{Q_{3 r}}\left|\theta\left(\boldsymbol{a}, Q_{3 r}\right)(x)\right|^{2} d x \leq \delta^{2},
$$

where $a(x, \xi)$ is zero extended from $Q_{3 r} \cap \Omega$ to $Q_{3 r}$, and the parameter $\delta>0$ will be specified later.

It is obvious that the boundary geometric structure condition (1.9) implies that $\Omega$ is a $(\delta, R)$-Reifenberg flat domain satisfying A-type domain, which leads to the following condition (cf. [9]):

$$
\sup _{0<r \leq R y \in \Omega} \sup _{y=\Omega} \frac{\left|Q_{r}(y)\right|}{\left|Q_{r}(y) \cap \Omega\right|} \leq \sup _{0<r \leq R y \in \Omega} \sup _{\left|B_{r}(y) \cap \Omega\right|} \leq\left(\frac{2 \sqrt{2}}{1-\delta}\right)^{n} \leq\left(\frac{16 \sqrt{2}}{7}\right)^{n} .
$$


In addition, the number $\delta$ can be selected as a small positive constant in a universal way so that it depends only on the basic structural constants like $n, v, \Lambda, \gamma_{1}, \gamma_{2}, \omega(\cdot)$ and the $\delta$-flatness on the geometric boundary, saying $0<\delta<\frac{1}{8}$.

Finally, we are ready to summarize our main result of this paper as follows.

Theorem 1.2 Let $u \in W_{0}^{1,2}(\Omega)$ be the weak solution to nonlinear elliptic equations (1.1) with nonlinearities $\boldsymbol{a}(\xi, x)$ satisfying ellipticity and growth (1.3). Assume that $p(\cdot)$ is a log-Hölder continuous function with (1.5) and (1.6), $(\boldsymbol{a}(\xi, x), \Omega)$ satisfies $\left(\delta, R_{0}\right)$-vanishing of codimension 1 with Assumption 1.1. Suppose

$$
|F|^{p(x)} \in L(t, q)(\Omega), \quad t>1, \quad q \in(0,+\infty],
$$

then, there exist small constant $\delta_{0}=\delta_{0}\left(\gamma_{1}, \gamma_{2}\right)>0$ such that for every $\delta \in\left(0, \delta_{0}\right]$, we have $|D u|^{p(x)} \in$ $L(t, q)(\Omega)$ with the estimate

$$
\left\||D u|^{p(x)}\right\|_{L(t, q)(\Omega)} \leq c\left(\left\||F|^{p(x)}\right\|_{L(t, q)(\Omega)}+1\right)^{\frac{\gamma_{2}}{\gamma_{1}}}
$$

where the constant $c$ depends only on $n, \gamma_{1}, \gamma_{2}, \nu, \Lambda, t, q, R_{0}, \Omega, \omega(\cdot),|\Omega|$ (except in the case $q=\infty$, where $c$ depends on $\left.n, \gamma_{1}, \gamma_{2}, \nu, \Lambda, t, R_{0}, \Omega, \omega(\cdot),|\Omega|\right)$.

Let us now recall recent investigation techniques on the Calderón-Zygmund theory of PDEs with discontinuous coefficients. We would also like to mention that at present there were mainly three kinds of main different arguments to study the Calderón-Zygmund theory of elliptic and parabolic problems with discontinuous coefficients, except an approach of classical singular integral operators and its commutators. One was Byun-Wang's geometrical argument [7, 8], who reached the Calderón-Zygmund estimates by way of the weak compactness, the Hardy-Littlewood maximal function and the modified Vitali covering lemma originally due to Safonov's fundamental work [25]. This method was also developed by Caffarelli and Peral in [12] to obtain $W_{l o c}^{1, p}$-estimates for solutions of a large class of elliptic problems. Secondly, Dong-Kim-Krylov in $[15,20]$ gave a unified approach of studying $L^{p}$ solvability for elliptic and parabolic problems due to the Fefferman-Stein theorem on sharp functions and the Hardy-Littlewood maximal function theorem, which is mainly based on the pointwise estimates of the sharp functions of the spatial derivative of solutions. The third technique was called large-M-inequality principle from Acerbi-Mingione's work [1, 2], which directly argued on certain Calderón-Zygmund-type covering arguments instead of the maximal function operator and other harmonic analysis techniques such as the good- $\lambda$-inequality.

In this article, we focus on considering the global Calderón-Zygmund type estimate for the gradient of weak solution with variable power in the framework of Lorentz spaces to nonlinear elliptic equations (1.1) with partially regular nonlinearities in a nonsmooth domains. Our key argument was inspired by Acerbi-Mingione and Baroni's papers [1, 2, 3, 4] and Byun-Kim's recent work [11]. Here, we make use of so-called large-M-inequality principle to prove global variable Lorentz estimate for the gradients of weak solution to (1.1) over a bounded Reifenberg flatness domain. Our main strategy is based on making use of the reverse Hölder inequality, appropriate covering and iteration arguments to obtain the measure of the super-level set of the gradient of its unique solution. We would like to remark that a key ingredient proving main Theorem concerning variable exponent is to use so-called perturbation approach by various local comparisons with these problems of constant local maximal and minimal exponents $p^{+}$and $p^{-}$, which also leads to an indispensable constant controlled by so-called log-Hölder continuous condition, see [27].

The rest of the paper is organized as follows. Section 2 is devoted to introduce some useful lemmas. In section 3 , we focus on proving our main theorem. 


\section{Technical tools}

In the section we present some useful lemmas, which will play essential roles in proving our main conclusion. Let us denote by $c(n, v, \Lambda, \cdots)$ a universal constant depending only on prescribed quantities and possibly varying from line to line in the context.

First, we need to make use of a fact that the elliptic equations considered is invariant under scaling and normalization, see Lemma 3.1 in [11].

Lemma 2.1 Fixed $K, \rho>0$, we define a normalization by

$$
\tilde{\boldsymbol{a}}(\xi, x)=\frac{\boldsymbol{a}(K \xi, \rho x)}{K}, \tilde{u}(x)=\frac{u(\rho x)}{K \rho}, \tilde{\boldsymbol{F}}(x)=\frac{\boldsymbol{F}(\rho x)}{K}
$$

and the set $\tilde{\Omega}=\left\{\frac{x}{\rho}: x \in \Omega\right\}$, then we have

(i) If $u$ is a weak solution of (1.1), then $\tilde{u}$ is also a weak solution of

$$
\begin{cases}\operatorname{div}(\tilde{\boldsymbol{a}}(D \tilde{u}, x)=\operatorname{div} \tilde{\boldsymbol{F}}, & \text { in } \tilde{\Omega}, \\ \tilde{u}=0, & \text { on } \partial \tilde{\Omega} .\end{cases}
$$

(ii) If the nonlinearity a satisfies assumption (1.3), then so dose $\tilde{\boldsymbol{a}}$ with the same constants $v, \Lambda$.

(iii) If the nonlinearity $(\boldsymbol{a}(\xi, x), \Omega)$ is $(\delta, R)$-vanishing of codimension 1 in $\Omega$, then $(\tilde{\boldsymbol{a}}(\xi, x), \Omega)$ is $\left(\delta, \frac{R}{\rho}\right)$ vanishing of codimension 1 in $\tilde{\Omega}$.

Secondly, let us collect some preliminary results concerning embedding relations involving the Lorentz spaces, which will be used in the sequel.

Proposition 2.2 Let $U$ be a bounded measurable subset of $\mathbb{R}^{n}$. Then the following holds:

(i) If $0<q_{1}, q_{2} \leq \infty$, and $1 \leq t_{1}<t_{2}<\infty$, then $L\left(t_{2}, q_{2}\right)(U) \subset L\left(t_{1}, q_{1}\right)(U)$ with the estimate

$$
\|g\|_{L\left(t_{1}, q_{1}\right)(U)} \leq c\left(t_{1}, t_{2}, q_{1}, q_{2}, U\right)\|g\|_{L\left(t_{2}, q_{2}\right)(U)} .
$$

(ii) If $1 \leq t<\infty$, and $0<q_{1}<q_{2} \leq \infty$, then $L\left(t, q_{1}\right)(U) \subset L\left(t, q_{2}\right)(U) \subset L(t, \infty)(U)$ with the estimate

$$
\|g\|_{L\left(t, q_{2}\right)(U)} \leq c\left(t, q_{1}, q_{2}\right)\|g\|_{L\left(t, q_{1}\right)(U)} .
$$

(iii) If $|g|^{\alpha} \in L(t, q)(U)$ for some $0<\alpha<\infty$, then $g \in L(\alpha t, \alpha q)(U)$ with the estimate

$$
\left\|\left.|| g\right|^{\alpha}\right\|_{L(t, q)(U)}=\|g\|_{L(\alpha t, \alpha q)(U)}^{\alpha} .
$$

The following two lemmas will play important roles in our main proof, which are indeed the variants of the classic Hardy's inequality and the reverse-Hölder inequality, respectively, see Lemma 3.4 and 3.5 in [3].

Lemma 2.3 Let $f:[0,+\infty) \rightarrow[0,+\infty)$ be a measurable function such that

$$
\int_{0}^{\infty} f(\lambda) d \lambda<\infty
$$

then, for any $\alpha \geq 1$ and $r>0$ there holds

$$
\int_{0}^{\infty} \lambda^{r}\left(\int_{\lambda}^{\infty} f(\mu) d \mu\right)^{\alpha} \frac{d \lambda}{\lambda} \leq\left(\frac{\alpha}{r}\right)^{\alpha} \int_{0}^{\infty} \lambda^{r}[\lambda f(\lambda)]^{\alpha} \frac{d \lambda}{\lambda} .
$$


Lemma 2.4 Let $h:[0,+\infty) \rightarrow[0,+\infty)$ be a non-increasing, measurable function. For $\alpha_{1} \leq \alpha_{2} \leq \infty$, $\alpha_{2}<\infty$ and $r>0$, then we have

$$
\left(\int_{\lambda}^{\infty}\left(\mu^{r} h(\mu)\right)^{\alpha_{2}} \frac{d \mu}{\mu}\right)^{\frac{1}{\alpha_{2}}} \leq \varepsilon \lambda^{r} h(\lambda)+\frac{c}{\varepsilon^{\frac{\alpha_{2}}{\alpha_{1}-1}}}\left(\int_{\lambda}^{\infty}\left(\mu^{r} h(\mu)\right)^{\alpha_{1}} \frac{d \mu}{\mu}\right)^{\frac{1}{\alpha_{1}}}
$$

with every $\varepsilon \in(0,1]$ and $\lambda \geq 0$. If $\alpha_{2}=\infty$, then it holds

$$
\sup _{\mu>\lambda}\left(\mu^{r} h(\mu)\right) \leq c \lambda^{r} h(\lambda)+c\left(\int_{\lambda}^{\infty}\left(\mu^{r} h(\mu)\right)^{\alpha_{1}} \frac{d \mu}{\mu}\right)^{\frac{1}{\alpha_{1}}},
$$

where the constant $c$ depends only on $\alpha_{1}, \alpha_{2}, r$ except in the case $\alpha_{2}=\infty$, in the case $c \equiv c\left(\alpha_{1}, r\right)$.

Also, we recall the well-known iteration argument, which can be found from Lemma 4.1 in [23].

Lemma 2.5 Let $\varphi:\left[r_{1}, 2 r_{1}\right] \rightarrow[0, \infty)$ be a function such that

$$
\varphi\left(\rho_{1}\right) \leq \frac{1}{2} \varphi\left(\rho_{2}\right)+B_{0}\left(\rho_{2}-\rho_{1}\right)^{-\beta}+L, \quad \forall r_{1}<\rho_{1}<\rho_{2}<2 r_{1},
$$

where $B_{0}, L \geq 0$ and $\beta>0$. Then

$$
\varphi\left(r_{1}\right) \leq c(\beta) B_{0} r_{1}^{-\beta}+c L
$$

Thirdly, let us show a higher integrability for the gradient of weak solution to nonlinear elliptic equations (1.1) in the Sobolev spaces $W_{0}^{1,2}(\Omega)$, which was proved by so-called reverse-Hölder inequality, see Chapter 5 in [18] or [22].

Lemma 2.6 Let $u \in W_{0}^{1,2}\left(Q_{2 r}\right)$ be a weak solution to nonlinear elliptic equations (1.1) under the assumptions (1.3), (1.5) and (1.6). If $|\boldsymbol{F}|^{p(x)} \in L^{p}\left(Q_{2 r}\right)$ for $p \geq 1$ and $r>0$ with $Q_{2 r} \subset \Omega$, then there exist constants $c=c\left(n, \gamma_{1}, \gamma_{2}, v, \Lambda\right)$ and $\sigma_{0}>0$ with

$$
\sigma_{0} \leq \frac{p \gamma_{1}}{2}-1
$$

such that for any $\sigma \leq \sigma_{0}$ we have

$$
\left(f_{Q_{r}}|D u|^{2(1+\sigma)} d x\right)^{\frac{1}{1+\sigma}} \leq c f_{Q_{2 r}}|D u|^{2} d x+c\left(f_{Q_{2 r}}|\boldsymbol{F}|^{2(1+\sigma)} d x\right)^{\frac{1}{1+\sigma}} .
$$

In addition, the following higher integrability on the boundary version is also a self-improving result due to the Reifenberg flatness domain belonging to A-type condition.

Lemma 2.7 Let $u \in W_{0}^{1,2}\left(\Omega_{2 r}\right)$ be a weak solution to nonlinear elliptic equations (1.1) with assumptions (1.3), (1.5) and (1.6). If $|\boldsymbol{F}|^{p(x)} \in L^{p}\left(\Omega_{2 r}\right)$ with $p \geq 1$ and $\Omega$ belongs to $\left(\delta, R_{0}\right)$-Reifenberg flat. For $0<r \leq \frac{R_{0}}{2}$ with

$$
Q_{2 r}^{+} \subset \Omega_{2 r} \subset Q_{2 r} \cap\left\{x_{n}>-4 \delta r\right\},
$$

then there exist constants $c=c\left(n, \gamma_{1}, \gamma_{2}, v, \Lambda\right)$ and $\sigma_{0}>0$ satisfying (2.7) such that for any $\sigma \leq \sigma_{0}$,

$$
\left(f_{\Omega_{r}}|D u|^{2(1+\sigma)} d x\right)^{\frac{1}{1+\sigma}} \leq c f_{\Omega_{2 r}}|D u|^{2} d x+c\left(f_{\Omega_{2 r}}|\boldsymbol{F}|^{2(1+\sigma)} d x\right)^{\frac{1}{1+\sigma}} .
$$


Proof. Without loss of generality, let $y \in \partial \Omega$ and $\Omega_{2 r}=\Omega_{2 r}(y)$. We also take $\varphi=\eta^{2} u$ in the neighborhood of boundary point. By using a similar procedure to Lemma 2.6 and the measure density property of $\Omega$, we see from the formula (1.11) and a zero extension of $u$ in $Q_{2 r}(y)$ that the conclusion is clearly true.

Finally, we are to focus on a few of comparison estimates in the interior point and boundary point. For simplicity, we set $y \in \Omega, r_{y}<\frac{R}{400}$ with any

$$
0<R \leq \min \left\{\frac{R_{0}}{2}, \frac{R_{0}}{c^{*}}, 1\right\},
$$

where $\left.c^{*}=c^{*}\left(n, \gamma_{1}, \gamma_{2}, v, \Lambda, \omega(\cdot),|\Omega|\right)\right) \geq|\Omega|+1$. For any fixed $x_{0} \in \Omega$, set

$$
\begin{gathered}
p^{-}=\inf _{\Omega_{2 R}\left(x_{0}\right)} p(x), p^{+}=\sup _{\Omega_{2 R}\left(x_{0}\right)} p(x) . \\
p_{y}^{-}=\inf _{\Omega_{200 r_{y}(y)}} p(x), p_{y}^{+}=\sup _{\Omega_{200 r_{y}}(y)} p(x) .
\end{gathered}
$$

For the interior case, we consider weak solution $u \in W^{1,2}\left(Q_{8}\right)$ of

$$
\operatorname{div} \mathbf{a}(D u, x)=\operatorname{div} \mathbf{F}, \quad \text { in } Q_{8} .
$$

Let $w$ be the weak solution of

$$
\begin{cases}\operatorname{div} \overline{\mathbf{a}}_{B_{7}^{\prime}}\left(D w, x^{1}\right)=0, & \text { in } Q_{7}, \\ w=v, & \text { on } \partial Q_{7} .\end{cases}
$$

We know that $\overline{\mathbf{a}}_{B_{7}^{\prime}}\left(\xi, x^{1}\right)$ is a Carathéodory vector valued function and satisfies ellipticity and growth condition (1.3). In what follows, let us recall some approximating estimates in accordance with the following comparisons from Byun and Kim's work, see [11].

Lemma 2.8 If $u$ is the weak solution of (2.11). Then for any $0<\epsilon<1$, there exists a constant $\delta=$ $\delta\left(n, \epsilon, \gamma_{1}, \gamma_{2}, v, \Lambda\right)$ such that

$$
f_{Q_{8}}|D u|^{2} d x \leq 1, f_{Q_{8}}|\boldsymbol{F}|^{2} d x \leq \delta^{\frac{\gamma_{1}}{\gamma_{2}}}, f_{Q_{8}}\left|\theta\left(\boldsymbol{a}, Q_{8}\right)\right|^{2} d x \leq \delta^{2}
$$

and if $w \in W^{1,2}\left(Q_{7}\right)$ is the weak solution of (2.12). Then

$$
f_{Q_{7}}|D u-D w|^{2} d x \leq \epsilon^{2},\|D w\|_{L^{\infty}\left(Q_{1}\right)}^{2} \leq c_{1}
$$

for some $c_{1}=c_{1}(n, v, \Lambda)$.

Proof. Similar to (5.11) and (5.19) in [11], we get

$$
\|D w\|_{L^{\infty}\left(Q_{1}\right)}^{2} \leq c_{1}
$$

and

$$
f_{Q_{7}}|D u-D w|^{2} d x \leq c\left(\delta^{\frac{\gamma_{1}}{\gamma_{2}}}+\delta^{\sigma_{1}}\right)
$$

for some positive constant $\sigma_{1}=\sigma_{1}(n, \nu, \Lambda)$. We choose $\delta>0$ small enough such that $\delta^{\frac{\gamma_{1}}{\gamma_{2}}}+\delta^{\sigma_{1}} \leq \epsilon^{2}$, which completes the proof. 
Now we study the boundary estimates for considering a weak solution $u \in W^{1,2}\left(\Omega_{8}\right)$ of

$$
\begin{cases}\operatorname{div} \mathbf{a}(D u, x)=\operatorname{div} \mathbf{F}, & \text { in } \Omega_{8} \\ u=0, & \text { on } \partial_{\omega} \Omega_{8}\end{cases}
$$

We first assume

$$
Q_{8}^{+} \subset \Omega_{8} \subset Q_{8} \cap\left\{x^{1}>-16 \delta\right\} .
$$

Consider a limiting problem in accordance with (2.16)

$$
\begin{cases}\operatorname{div} \overline{\mathbf{a}}_{B_{7}^{\prime}}\left(D h, x^{1}\right)=0, & \text { in } Q_{7}^{+}, \\ h=w, & \text { on } \partial T_{7},\end{cases}
$$

we obtain the boundary comparison estimate and Lipschitz boundedness for weak solution of the limiting problem (2.17) for details see Lemma 5.9 in [11] and the references therein.

Lemma 2.9 If $u$ is the weak solution of (2.15). Then for any $0<\epsilon<1$ and $\lambda \geq 1$ there exists a constant $\delta=\delta\left(n, \epsilon, \gamma_{1}, \gamma_{2}, v, \Lambda\right)$ such that

$$
f_{\Omega_{8}}|D u|^{2} d x \leq 1, \quad f_{\Omega_{8}}|\boldsymbol{F}|^{2} d x \leq \delta^{\frac{\gamma_{1}}{\gamma_{2}}}, f_{Q_{8}}\left|\theta\left(\boldsymbol{a}, Q_{8}\right)\right|^{2} d x \leq \delta^{2}
$$

and (2.16) hold; if $h \in W^{1,2}\left(Q_{7}^{+}\right)$is the weak solution of (2.17). Then for some constant $c_{2}=c_{2}(n, v, \Lambda)$

$$
f_{\Omega_{7}}|D u-D \bar{h}|^{2} d x \leq \epsilon^{2}, \quad\|D \bar{h}\|_{L^{\infty}\left(\Omega_{1}\right)}^{2} \leq c_{2}
$$

where $\bar{h}$ is the zero extension of $h$ from $Q_{7}^{+}$to $Q_{7}$.

\section{Proof of Theorem 1.2}

This section is devoted to proving Theorem 1.2 via the so-called large-M-inequality principle introduced by Acerbi-Mingione in [2]. We part it in six steps to prove it. In Step 1, for given $\lambda_{0}$ in (3.1) we show the Calderón-Zygmund type covering on the super-level set $E\left(\lambda, \Omega_{R}\left(x_{0}\right)\right)$, and establish the estimates of $\Omega_{r_{y}}(y)$. In Step 2, we give various comparison estimates. In Step 3, we employ the Vitali's covering argument to obtain estimate of the super-levels for the distribution with $E\left(\lambda, \Omega_{R}\left(x_{0}\right)\right)$. In Step 4 and Step 6, we get the conclusions, respectively, for $q<\infty$ and $q=\infty$ under a priori assumption $\left\||D u|^{p(x)}\right\|_{L(t, q)\left(\Omega_{2 R}\right)}<\infty$, which will be proved in Step 5.

Proof. We here only treat the boundary case. For the interior case, one can prove it by using similar but a much simple way, which the ideas and techniques are used for the boundary case. For the boundary case, we notice that the related qualities are still invariant by a proper translation and rotation of the original coordinates. For this, we keep using the same notations. Without loss of generality, we may assume that $R_{0} \leq 1$ by a scaling transformation in Assumption 1.1.

Step 1. Let $u$ be weak solution of (1.1). For any fixed $x_{0} \in \Omega$, we define the quantity:

$$
\lambda_{0}:=f_{\Omega_{2 R}\left(x_{0}\right)}|D u|^{\frac{2 p(x)}{p^{-}}} d x+\frac{1}{\delta}\left(f_{\Omega_{2 R}\left(x_{0}\right)}\left(|\mathbf{F}|^{\frac{2 p(x)}{p^{-}}}+1\right)^{\eta} d x\right)^{\frac{1}{\eta}},
$$

where $\delta>0$ and $\eta>1$ will be specified later. Introducing the super-level set

$$
E\left(\lambda, \Omega_{R}\left(x_{0}\right)\right):=\left\{x \in \Omega_{R}\left(x_{0}\right),|D u|^{\frac{2 p(x)}{p^{-}}}>\lambda\right\}
$$


for $\lambda>M \lambda_{0} \geq 1$ with $M=\left(\frac{12800 \sqrt{2}}{7}\right)^{n}$. Taking a point $y \in E\left(\lambda, \Omega_{R}\right)$, for radii $0<r \leq R$ we let

$$
C Z\left(\Omega_{r}(y)\right):=f_{\Omega_{r}(y)}|D u|^{\frac{2 p(x)}{p^{-}}} d x+\frac{1}{\delta}\left(f_{\Omega_{r}(y)}|\mathbf{F}|^{\frac{2 p(x)}{p^{-}} \eta} d x\right)^{\frac{1}{\eta}} .
$$

Simply enlarging the domain of the integration yields that for $\frac{R}{400} \leq r \leq R$,

$$
\begin{aligned}
& C Z\left(\Omega_{r}(y)\right) \\
\leq & \frac{\left|\Omega_{2 R}\left(x_{0}\right)\right|}{\left|\Omega_{r}(y)\right|} f_{\Omega_{2 R}\left(x_{0}\right)}|D u|^{\frac{2 p(x)}{p^{-}}} d x+\left(\frac{\left|\Omega_{2 R}\left(x_{0}\right)\right|}{\left|\Omega_{r}(y)\right|}\right)^{\frac{1}{\eta}} \frac{1}{\delta}\left(f_{\Omega_{2 R}\left(x_{0}\right)}|\mathbf{F}|^{\frac{2 p(x)}{p^{-}} \eta} d x\right)^{\frac{1}{\eta}} \\
\leq & \frac{\left|\Omega_{2 R}\left(x_{0}\right)\right|}{\left|\Omega_{r}(y)\right|}\left(f_{\Omega_{2 R}\left(x_{0}\right)}|D u|^{\frac{2 p(x)}{p^{-}}} d x+\frac{1}{\delta}\left(f_{\Omega_{2 R}\left(x_{0}\right)}|\mathbf{F}|^{\frac{2 p(x)}{p^{-}} \eta} d x\right)^{\frac{1}{\eta}}\right) \\
\leq & \frac{\left|B_{2 R}\left(x_{0}\right)\right|}{\left|B_{r}(y)\right|} \frac{\left|B_{r}(y)\right|}{\left|\Omega_{r}(y)\right|} \lambda_{0} \\
\leq & \left(\frac{2 R}{r}\right)^{n}\left(\frac{16 \sqrt{2}}{7}\right)^{n} \lambda_{0} \\
\leq & \left(\frac{12800 \sqrt{2}}{7}\right)^{n} \lambda_{0}<\lambda .
\end{aligned}
$$

This means that in the setting of $\frac{R}{400} \leq r \leq R$ one has

$$
C Z\left(\Omega_{r}(y)\right)<\lambda
$$

On the other hand, by Lebesgue's differentiation theorem we get that for small radii $0<r<<1$,

$$
C Z\left(\Omega_{r}(y)\right)>\lambda
$$

Therefore, according to the absolutely continuity of the integral w. r. t. its domain, we can pick the maximal radius $r_{y}$ such that

$$
C Z\left(\Omega_{r_{y}}(y)\right)=f_{\Omega_{r_{y}}(y)}|D u|^{\frac{2 p(x)}{p^{-}}} d x+\frac{1}{\delta}\left(f_{\Omega_{r_{y}}(y)}|\mathbf{F}|^{\frac{2 p(x)}{p^{-}} \eta} d x\right)^{\frac{1}{\eta}}=\lambda
$$

for $y \in E\left(\lambda, \Omega_{R}\left(x_{0}\right)\right)$. Moreover, for any $r \in\left(r_{y}, R\right]$ one has

$$
C Z\left(\Omega_{r}\left(x_{y}\right)\right)<\lambda .
$$

By (3.4) we then have the following alternatives:

$$
\frac{\lambda}{2} \leq f_{\Omega_{r_{y}}(y)}|D u|^{\frac{2 p(x)}{p^{-}}} d x \quad \text { or } \quad\left(\frac{\delta \lambda}{2}\right)^{\eta} \leq f_{\Omega_{r_{y}}(y)}|\mathbf{F}|^{\frac{2 p(x)}{p^{-}} \eta} d x .
$$

First, we consider the first case in (3.6), and split this integral average as follows:

$$
\begin{aligned}
& f_{\Omega_{r_{y}}(y)}|D u|^{\frac{2 p(x)}{p^{-}}} d x \\
\leq & \frac{\left|\Omega_{r_{y}}(y) \backslash E\left(\frac{\lambda}{4}, \Omega_{2 R}\left(x_{0}\right)\right)\right|}{\left|\Omega_{r_{y}}(y)\right|} f_{\Omega_{r_{y}}(y) \backslash E\left(\frac{\lambda}{4}, \Omega_{2 R}\left(x_{0}\right)\right)}|D u|^{\frac{2 p(x)}{p^{-}}} d x+\frac{1}{\left|\Omega_{r_{y}}(y)\right|} \int_{\Omega_{r_{y}}(y) \cap E\left(\frac{\lambda}{4}, \Omega_{2 R}\left(x_{0}\right)\right)}|D u|^{\frac{2 p(x)}{p^{-}}} d x
\end{aligned}
$$




$$
\begin{aligned}
& \leq \frac{\lambda}{4}+c \frac{\left|\Omega_{r_{y}}(y) \cap E\left(\frac{\lambda}{4}, \Omega_{2 R}\left(x_{0}\right)\right)\right|}{\left|\Omega_{r_{y}}(y) \cap E\left(\frac{\lambda}{4}, \Omega_{2 R}\left(x_{0}\right)\right)\right|^{\frac{1}{1+\sigma^{\prime}}}} \frac{1}{\left|\Omega_{r_{y}}(y)\right|}\left(\int_{\Omega_{r_{y}}(y)}|D u|^{\frac{2 p(x)}{p^{-}}\left(1+\sigma^{\prime}\right)} d x\right)^{\frac{1}{1+\sigma^{\prime}}} \\
& \leq \frac{\lambda}{4}+c\left(\frac{\left|\Omega_{r_{y}}(y) \cap E\left(\frac{\lambda}{4}, \Omega_{2 R}\left(x_{0}\right)\right)\right|}{\left|\Omega_{r_{y}}(y)\right|}\right)^{1-\frac{1}{1+\sigma^{\prime}}}\left(f_{\Omega_{r_{y}}(y)}|D u|^{\frac{2 p(x)}{p^{-}}\left(1+\sigma^{\prime}\right)} d x\right)^{\frac{1}{1+\sigma^{\prime}}} .
\end{aligned}
$$

Let us take

$$
0<\sigma^{\prime} \leq \frac{\gamma_{1}(1+\sigma)}{\gamma_{1}+\omega(2 R)}-1
$$

with $\sigma$ as the same of Lemma 2.7, it yields the following inequality

$$
\frac{p(x)}{p^{-}}\left(1+\sigma^{\prime}\right)=\left(1+\frac{p(x)-p^{-}}{p^{-}}\right)\left(1+\sigma^{\prime}\right) \leq\left(1+\frac{\omega(2 R)}{p^{-}}\right)\left(1+\sigma^{\prime}\right) \leq(1+\sigma) .
$$

Thus, by taking $\eta=1+\sigma^{\prime}$ with (3.5) we obtain

$$
\begin{aligned}
& \left(\int_{\Omega_{r y}(y)}|D u|^{\frac{2 p(x)}{p^{-}}\left(1+\sigma^{\prime}\right)} d x\right)^{\frac{1}{1+\sigma^{\prime}}} \\
\leq & c\left(\int_{\Omega_{2 r_{y}}(y)}|D u|^{\frac{2 p(x)}{p^{-}}} d x+\left(f_{\Omega_{2 r y}(y)}|\mathbf{F}|^{\frac{2 p(x)}{p^{-}}\left(1+\sigma^{\prime}\right)} d x\right)^{\frac{1}{1+\sigma^{\prime}}}+1\right) \\
\leq & c \lambda,
\end{aligned}
$$

where the first inequality is due to the reverse Hölder inequality shown by lemma 2.7 and (1.6). Therefore, using (3.6) and reabsorbing

$$
\frac{\lambda}{4} \leq c\left(\frac{\left|\Omega_{r_{y}}(y) \cap E\left(\frac{\lambda}{4}, \Omega_{2 R}\left(x_{0}\right)\right)\right|}{\left|\Omega_{r_{y}}(y)\right|}\right)^{1-\frac{1}{1+\sigma^{\prime}}} \lambda,
$$

which implies

$$
\left|\Omega_{r_{y}}(y)\right| \leq c\left|\Omega_{r_{y}}(y) \cap E\left(\frac{\lambda}{4}, \Omega_{2 R}\left(x_{0}\right)\right)\right|
$$

with the constant $c$ depending only on $n, \gamma_{2}, \gamma_{2}, \nu, \Lambda, t$.

To the second estimate of (3.6), by taking $\zeta=\frac{\delta}{4}$, Fubini's theorem and splitting the integral we get

$$
\begin{aligned}
& \left(\frac{\lambda \delta}{2}\right)^{\eta} \leq f_{\Omega_{r_{y}}(y)}|\mathbf{F}|^{\frac{2 p(x)}{p^{-}} \eta} d x \\
= & \frac{\eta}{\left|\Omega_{r_{y}}(y)\right|} \int_{0}^{\infty} \mu^{\eta}\left|\left\{x \in \Omega_{r_{y}}(y):|\mathbf{F}|^{\frac{2 p(x)}{p^{-}}}>\mu\right\}\right| \frac{d \mu}{\mu} \\
= & \frac{\eta}{\left|\Omega_{r_{y}}(y)\right|} \int_{0}^{\zeta \lambda} \mu^{\eta}\left|\left\{x \in \Omega_{r_{y}}(y):|\mathbf{F}|^{\frac{2 p(x)}{p^{-}}}>\mu\right\}\right| \frac{d \mu}{\mu}+\frac{\eta}{\left|\Omega_{r_{y}}(y)\right|} \int_{\zeta \lambda}^{\infty} \mu^{\eta}\left|\left\{x \in \Omega_{r_{y}}(y):|\mathbf{F}|^{\frac{2 p(x)}{p^{-}}}>\mu\right\}\right| \frac{d \mu}{\mu} \\
\leq & (\zeta \lambda)^{\eta}+\frac{\eta}{\left|\Omega_{r_{y}}(y)\right|} \int_{\zeta \lambda}^{\infty} \mu^{\eta}\left|\left\{x \in \Omega_{r_{y}}(y):|\mathbf{F}|^{\frac{2 p(x)}{p^{-}}}>\mu\right\}\right| \frac{d \mu}{\mu} .
\end{aligned}
$$

Let $\delta=4 \zeta$, then the first term above on the right-hand side can be reabsorbed

$$
(\zeta \lambda)^{\eta} \leq \frac{\eta}{\left|\Omega_{r_{y}}(y)\right|} \int_{\zeta \lambda}^{\infty} \mu^{\eta}\left|\left\{x \in \Omega_{r_{y}}(y):|\mathbf{F}|^{\frac{2 p(x)}{p^{-}}}>\mu\right\}\right| \frac{d \mu}{\mu},
$$

which yields

$$
\left|\Omega_{r_{y}}(y)\right| \leq \frac{\eta}{(\zeta \lambda)^{\eta}} \int_{\zeta \lambda}^{\infty} \mu^{\eta}\left|\left\{x \in \Omega_{r_{y}}(y):|\mathbf{F}|^{\frac{2 p(x)}{p^{-}}}>\mu\right\}\right| \frac{d \mu}{\mu}
$$


Putting (3.7) and (3.8) together leads to

$$
\left|\Omega_{r_{y}}(y)\right| \leq c\left|\Omega_{r_{y}}(y) \cap E\left(\frac{\lambda}{4}, \Omega_{2 R}\left(x_{0}\right)\right)\right|+\frac{c \eta}{(\zeta \lambda)^{\eta}} \int_{\zeta \lambda}^{\infty} \mu^{\eta}\left|\left\{x \in \Omega_{r_{y}}(y):|\mathbf{F}|^{\frac{2 p(x)}{p^{-}}}>\mu\right\}\right| \frac{d \mu}{\mu} .
$$

Step 2. Since $\Omega$ is $\left(\delta, R_{0}\right)$-Reifenberg flat for some $R_{0}>0$ shown as Assumption 1.1, we have

$$
f_{Q_{200 r_{y}(y)}}\left|\theta\left(\mathbf{a}, Q_{200 r_{y}}(y)\right)\right|^{2} d x \leq \delta^{2}
$$

Taking into account (2.18) and (3.5) yields

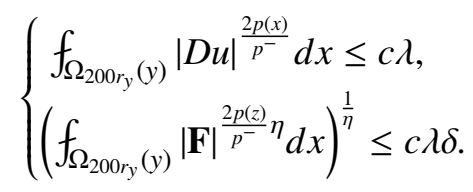

In what follows, it suffices to show that

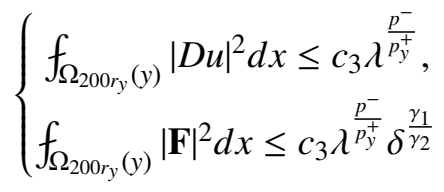

for some constant $c_{3} \geq 1$. We first obverse that

$$
\left(f_{\Omega_{200 r_{y}(y)}}|D u|^{2} d x\right)^{p_{y}^{+}-p_{y}^{-}} \leq c .
$$

where $c \geq 1$ is a universal constant. Note that $|\mathbf{F}|^{p(x)} \in L(t, q)(\Omega) \subset L^{1}(\Omega)$ for $t>1$ and $0<q \leq \infty$, which implies $\mathbf{F} \in L^{p(x)}(\Omega)$. Considering $\max \left\{\left(\int_{\Omega}|\mathbf{F}|^{p(x)} d x\right)^{\frac{1}{\gamma_{1}}},\left(\int_{\Omega}|\mathbf{F}|^{p(x)} d x\right)^{\frac{1}{\gamma_{2}}}\right\} \leq\|\mathbf{F}\|_{L^{p(x)}}$ by Corollary 2.23 in [13], which yields $\int_{\Omega}|\mathbf{F}|^{p(x)} d x \leq c$. This together with $L^{2}$-estimate leads to that

$$
\int_{\Omega}|\mathbf{F}|^{2} d x \leq \int_{\Omega}\left(|\mathbf{F}|^{p(x)}+1\right) d x \leq c+|\Omega|,
$$

and

$$
\int_{\Omega}|D u|^{2} d x \leq c \int_{\Omega}|\mathbf{F}|^{2} d x \leq c(1+|\Omega|) .
$$

Note that $p_{y}^{+}-p_{y}^{-} \leq \omega\left(400 r_{y}\right)$ it yields that

$$
\begin{aligned}
& \left(f_{\left.\Omega_{200 r_{y}(y)}|D u|^{2} d x\right)^{p_{y}^{+}-p_{y}^{-}}}\right)_{=}\left(\frac{1}{\left|\Omega_{200 r_{y}}(y)\right|}\right)^{p_{y}^{+}-p_{y}^{-}}\left(\int_{\left.\Omega_{200 r_{y}(y)}|D u|^{2} d x\right)^{p_{y}^{+}-p_{y}^{-}}}\right. \\
\leq & c\left(\frac{1}{\left|Q_{400 r_{y}}(y)\right|}\right)^{p_{y}^{+}-p_{y}^{-}}\left(\int_{\Omega_{200 r_{y}}(y)}|D u|^{2} d x\right)^{p_{y}^{+}-p_{y}^{-}} \\
\leq & c\left(\frac{1}{400 r_{y}}\right)^{n \omega\left(400 r_{y}\right)}\left(\int_{\left.\Omega_{200 r_{y}(y)}|D u|^{2} d x\right)^{p_{y}^{+}-p_{y}^{-}}}\right.
\end{aligned}
$$




$$
\leq c\left(\int_{\Omega_{200 r y}(y)}|D u|^{2} d x\right)^{p_{y}^{+}-p_{y}^{-}} .
$$

On the other hand, by using (3.15) and $\frac{1}{400 r_{y}} \geq \frac{1}{R} \geq \frac{c^{*}}{R_{0}} \geq|\Omega|+1$ with (2.10) we find that

$$
\begin{aligned}
& \left(\int_{\Omega_{200 r_{y}}(y)}|D u|^{2} d x\right)^{p_{y}^{+}-p_{y}^{-}} \\
\leq & \left(\int_{\Omega}|D u|^{2} d x\right)^{p_{y}^{+}-p_{y}^{-}} \\
\leq & c(|\Omega|+1)^{p_{y}^{+}-p_{y}^{-}} \\
\leq & c\left(\frac{1}{400 r_{y}}\right)^{\omega\left(400 r_{y}\right)} \leq c,
\end{aligned}
$$

which prove (3.13) due to (1.6). Recalling $\gamma_{1} \leq p_{y}^{-}$and (3.13) with $\lambda>1$, we obtain

$$
\begin{aligned}
& f_{\Omega_{200 r y}(y)}|D u|^{2} d x \\
& =\left(f_{\Omega_{200 r_{y}(y)}}|D u|^{2} d x\right)^{\frac{p_{y}^{+}-p_{y}^{-}}{p_{y}^{+}}} \cdot\left(f_{\Omega_{200 r y}(y)}|D u|^{2} d x\right)^{\frac{p_{y}^{-}}{p_{y}^{+}}} \\
& \leq c^{\frac{1}{\gamma_{1}}}\left(f_{\Omega_{200 r_{y}(y)}}|D u|^{2} d x\right)^{\frac{p_{y}^{-}}{p_{y}^{+}}} \\
& \leq c\left(\int_{\Omega_{200 r_{y}(y)}}|D u|^{\frac{2 p_{y}^{-}}{p^{-}}} d x\right)^{\frac{p^{-}}{p_{y}^{+}}} \\
& \leq c\left(\int_{\Omega_{200 r_{y}(y)}}|D u|^{\frac{2 p(x)}{p^{-}}} d x+1\right)^{\frac{p^{-}}{p_{y}^{+}}} \leq c \lambda^{\frac{p^{-}}{p_{y}^{+}}} .
\end{aligned}
$$

Similarly, recalling $\delta \lambda_{0} \geq 1$ and $\lambda \geq M \lambda_{0}$ we find that

$$
\begin{aligned}
f_{\Omega_{200 r y}(y)}|\mathbf{F}|^{2} d x & \leq c\left(f_{\Omega_{200 r_{y}(y)}}|\mathbf{F}|^{\frac{2 p(x)}{p^{-}}} d x+1\right)^{\frac{p^{-}}{p_{y}^{+}}} \\
& \leq c(\delta \lambda+1)^{\frac{p^{-}}{p_{y}^{+}}} \\
& \leq c\left(\delta \lambda+\delta \lambda_{0}\right)^{\frac{p^{-}}{p_{y}^{+}}} \leq c \lambda^{\frac{p^{-}}{p_{y}^{+}}} \delta^{\frac{\gamma_{1}}{\gamma_{2}}} .
\end{aligned}
$$

Now we define

$$
\tilde{\mathbf{a}}_{y}\left(D \tilde{u}_{y}, x\right)=\frac{\mathbf{a}\left(D u, 25 r_{y} x\right)}{\sqrt{c_{3} \lambda^{\frac{p^{-}}{p_{y}^{+}}}}}, \quad \tilde{u}_{y}(x)=\frac{u\left(25 r_{y} x\right)}{25 r_{y} \sqrt{c_{3} \lambda^{\frac{p_{y}^{+}}{p_{y}^{+}}}}}, \quad \tilde{\mathbf{F}}_{y}(x)=\frac{\mathbf{F}\left(25 r_{y} x\right)}{\sqrt{c_{3} \lambda^{\frac{p^{-}}{p_{y}^{+}}}}} .
$$

By Lemma 2.1, we get that $\tilde{u}_{y}$ is a weak solution of

$$
\begin{cases}\operatorname{div}\left(\tilde{\mathbf{a}}_{y}\left(D \tilde{u}_{y}, x\right)=\operatorname{div} \tilde{\mathbf{F}}_{y},\right. & \text { in } \Omega_{8}, \\ \tilde{u}_{y}=0, & \text { on } \partial \Omega_{8} .\end{cases}
$$


Moreover, using (3.10) and (3.12) leads to

$$
\left\{\begin{array}{l}
f_{Q_{8}}\left|\theta\left(\tilde{\mathbf{a}}_{y}, Q_{8}\right)\right|^{2} d x \leq \delta^{2}, \\
f_{\Omega_{8}}\left|D \tilde{u}_{y}\right|^{2} d x \leq 1 \\
f_{\Omega_{8}}\left|\tilde{\mathbf{F}}_{y}\right|^{2} d x \leq \delta^{\frac{\gamma_{1}}{\gamma_{2}}}
\end{array}\right.
$$

Thus, by Lemma 2.9 it follows that

$$
f_{\Omega_{7}}\left|D \tilde{u}_{y}-D \tilde{h}_{y}\right|^{2} d x \leq \epsilon^{2},
$$

and

$$
\left\|\nabla \tilde{h}_{y}\right\|_{L^{\infty}\left(\Omega_{1}\right)} \leq c_{2}
$$

We now scale back by

$$
\tilde{h}_{y}(x)=\frac{\bar{h}_{y}\left(25 r_{y} x\right)}{25 r_{y} \sqrt{c_{3} \lambda^{\frac{p^{-}}{p_{y}^{+}}}}},
$$

where $\bar{h}_{y}$ is the weak solution of (2.17) replacing $Q_{7}^{+}, T_{4}$ by $Q_{175 r_{y}}^{+}(y), T_{175 r_{y}}(y)$, respectively. By extending the weak solution by zero from $Q_{175 r_{y}}^{+}(y)$ to $\Omega_{175 r_{y}}(y)$ it yields

$$
f_{\Omega_{175 r_{y}(y)}}\left|D u-D \bar{h}_{y}\right|^{2} d x \leq c_{3} \lambda^{\frac{p^{-}}{p_{y}^{+}}} \epsilon^{2}
$$

and

$$
\left\|\nabla \bar{h}_{y}\right\|_{L^{\infty}\left(\Omega_{25 r y}(y)\right)} \leq c_{2} \lambda^{\frac{p^{-}}{p_{y}^{+}}} .
$$

For the case of interior estimates, similar to (3.17) and (3.18) we have

$$
f_{Q_{175 r_{y}(y)}}\left|D u-D w_{y}\right|^{2} d x \leq c_{4} \lambda^{\frac{p^{-}}{p_{y}^{+}}} \epsilon^{2}
$$

and

$$
\left\|D w_{y}\right\|_{L^{\infty}\left(Q_{25 r_{y}}(y)\right)} \leq c_{1} \lambda^{\frac{p_{y}^{-}}{p_{y}^{+}}}
$$

where $w_{y}$ is any weak solution of (2.12) replacing $Q_{7}$ by $Q_{175 r_{y}}(y)$.

Step 3. For any fixed point $x \in \Omega$, we select a universal constant $R=R\left(n, \gamma_{1}, \gamma_{2}, v, \Lambda, \omega(\cdot), R_{0}\right)>0$ so that the prescribed condition (2.10) holds true. Furthermore, there exists a constant $\delta=\delta\left(n, \epsilon, \gamma_{1}, \gamma_{2}, v, \Lambda\right)>0$ such that lemma 2.8 and 2.9 hold, we write

$$
c_{0}=\max \left\{c_{1}, c_{2}, 1\right\} .
$$

For any $x \in E\left(A \lambda, \Omega_{R}\left(x_{0}\right)\right)$, we consider the collection $\mathcal{B}_{\lambda}$ of all subset $\Omega_{r_{y}}(y)$. By the Vitali-type covering argument, we extract a countable sub-collection $\left\{\Omega_{r_{i}}\left(y_{i}\right)\right\} \in \mathcal{B}_{\lambda}$, such that 5-times enlarged balls $\Omega_{5 r_{i}}\left(y_{i}\right)$ cover almost all $E\left(A \lambda, \Omega_{R}\left(x_{0}\right)\right)$ and the balls $\left\{\Omega_{r_{i}}\left(y_{i}\right)\right\}_{i=1}^{\infty}$ are pointwise disjoints with $y_{i} \in E\left(A \lambda, \Omega_{R}\left(x_{0}\right)\right), r_{i}=$ $r_{y_{i}}$ for $i \in \mathbb{N}$, and we have the following relation

$$
\Omega_{r_{i}}\left(y_{i}\right) \cap \Omega_{r_{j}}\left(y_{j}\right)=\emptyset, \quad \text { whenever } i \neq j \text {, and } E\left(A \lambda, \Omega_{R}\left(x_{0}\right)\right) \subset \underset{i \in \mathbb{N}}{\cup} \Omega_{5 r_{i}}\left(y_{i}\right) \cup \mathcal{N}_{\lambda}
$$


with $\left|\mathcal{N}_{\lambda}\right|=0$. Let $A=\left(4 c_{0}\right)^{\frac{\gamma_{2}}{\gamma_{1}}}$, then

$$
E\left(A \lambda, \Omega_{R}\left(x_{0}\right)\right) \subset \cup \Omega_{i \geq 1} \Omega_{5 r_{i}}\left(y_{i}\right) \cup \mathcal{N}_{\lambda} .
$$

By (3.22), we separate the resulting estimation into the interior and boundary cases to derive that

$$
\begin{aligned}
& \left|E(A \lambda), \Omega_{R}\left(x_{0}\right)\right|=\left|E\left(\left(4 c_{0}\right)^{\frac{\gamma_{2}}{\gamma_{1}}} \lambda\right), \Omega_{R}\left(x_{0}\right)\right| \\
= & \left|\left\{x \in \Omega_{R}\left(x_{0}\right):|D u|^{\frac{2 p(x)}{p^{-}}} \geq\left(4 c_{0}\right)^{\frac{\gamma_{2}}{\gamma_{1}}} \lambda\right\}\right| \\
\leq & \sum_{i \geq 1}\left|\left\{x \in \Omega_{5 r_{i}}\left(y_{i}\right):|D u|^{2} \geq 4 c_{0} \lambda^{\frac{p^{-}}{p(x)}}\right\}\right| \\
\leq & \sum_{i \geq 1}\left|\left\{x \in \Omega_{5 r_{i}}\left(y_{i}\right):|D u|^{2} \geq 4 c_{0} \lambda^{\frac{p^{-}}{p(x)}}\right\}\right| \\
= & \sum_{\text {interior case }}\left|\left\{x \in \Omega_{5 r_{i}}\left(y_{i}\right):|D u|^{2} \geq 4 c_{0} \lambda^{\frac{p^{-}}{p(x)}}\right\}\right| \\
& +\sum_{\text {boundary case }}\left|\left\{x \in \Omega_{5 r_{i}}\left(y_{i}\right):|D u|^{2} \geq 4 c_{0} \lambda^{\frac{p^{-}}{p(x)}}\right\}\right| .
\end{aligned}
$$

Next, we denote $w_{i}=w_{y_{i}}, \bar{h}_{i}=\bar{h}_{y_{i}}, p_{i}^{+}=p_{y_{i}}^{+}$for the sake of simplicity. For the interior case, from (3.19), (3.20) and (3.21) we get that

$$
\begin{aligned}
& \left|\left\{x \in \Omega_{5 r_{i}}\left(y_{i}\right):|D u|^{2} \geq 4 c_{0} \lambda^{\frac{p^{-}}{p(x)}}\right\}\right| \\
\leq & \left|\left\{x \in Q_{5 r_{i}}\left(y_{i}\right):|D u|^{2} \geq 4 c_{1} \lambda^{\frac{p^{-}}{p(x)}}\right\}\right| \\
\leq & \left|\left\{x \in Q_{5 r_{i}}\left(y_{i}\right):\left|D u-D w_{i}\right|^{2} \geq c_{1} \lambda^{\frac{p^{-}}{p_{i}^{+}}}\right\}\right| \\
& +\left|\left\{x \in Q_{5 r_{i}}\left(y_{i}\right):\left|D w_{i}\right|^{2} \geq c_{1} \lambda^{\frac{p^{-}}{p_{i}^{+}}}\right\}\right| \\
\leq & \frac{1}{{\frac{p^{-}}{p^{+}}}_{p_{i}^{+}}} \int_{Q_{5 r_{i}}\left(y_{i}\right)}\left|D u-D w_{i}\right|^{2} d x \\
\leq & c \epsilon^{2}\left|Q_{5 r_{i}}\left(y_{i}\right)\right| \leq c \epsilon^{2}\left|Q_{r_{i}}\left(y_{i}\right)\right|,
\end{aligned}
$$

where we used the weak $(1,1)$-type estimate:

$$
|\{x \in E: f(x)>\lambda\}| \leq \frac{1}{\lambda} \int_{E} f(x) d x .
$$

Similarly, for the boundary case we also obtain that

$$
\begin{array}{cc} 
& \left|\left\{x \in \Omega_{5 r_{i}}\left(y_{i}\right):|D u(x)|^{2} \geq 4 c_{0} \lambda^{\frac{p^{-}}{p(x)}}\right\}\right| \\
= & \left|\left\{z \in \Omega_{5 r_{i}}\left(y_{i}\right):|D u(z)|^{2} \geq 4 c_{0} \lambda^{\frac{p^{-}}{p(z)}}\right\}\right| \\
\leq & \left|\left\{z \in \Omega_{25 r_{i}}\left(y_{i}\right):|D u(z)|^{2} \geq 4 c_{2} \lambda^{\frac{p^{-}}{p(z)}}\right\}\right| \\
\leq & \left|\left\{z \in \Omega_{25 r_{i}}\left(y_{i}\right):\left|D u-D \bar{h}_{i}\right|^{2} \geq c_{2} \lambda^{\frac{p^{-}}{p_{i}^{+}}}\right\}\right| \\
& +\left|\left\{z \in \Omega_{25 r_{i}}\left(y_{i}\right):\left|D \bar{h}_{i}\right|^{2} \geq c_{2} \lambda^{\frac{p^{-}}{p_{i}^{+}}}\right\}\right|
\end{array}
$$




$$
\begin{aligned}
& \leq \frac{1}{c_{2} \lambda^{\frac{p^{-}}{p_{i}^{+}}}} \int_{\Omega_{25 r_{i}}\left(y_{i}\right)}\left|D u-D \bar{h}_{i}\right|^{2} d z \\
& \leq c \epsilon^{2}\left|\Omega_{175 r_{i}}\left(y_{i}\right)\right| \leq c \epsilon^{2}\left|Q_{175 r_{i}}\left(y_{i}\right)\right|=c \epsilon^{2}\left|Q_{r_{i}}\left(y_{i}\right)\right| \leq c \epsilon^{2} \frac{\left|Q_{r_{i}}\left(y_{i}\right)\right|}{\left|\Omega_{r_{i}}\left(y_{i}\right)\right|}\left|\Omega_{r_{i}}\left(y_{i}\right)\right| \\
& \leq c \epsilon^{2}\left(\frac{2 \sqrt{2}}{1-\delta}\right)^{n}\left|\Omega_{r_{i}}\left(y_{i}\right)\right| \leq c \epsilon^{2}\left(\frac{16 \sqrt{2}}{7}\right)^{n}\left|\Omega_{r_{i}}\left(y_{i}\right)\right| .
\end{aligned}
$$

We now combine (3.23), (3.24) and (3.25) to derive

$$
\left|E\left(A \lambda, \Omega_{R}\left(x_{0}\right)\right)\right| \leq c \epsilon^{2} \sum_{i \geq 1}\left|\Omega_{r_{i}}\left(y_{i}\right)\right| .
$$

Using the Vitali-type covering argument and (3.9), we conclude that

$$
\begin{aligned}
& \left|E\left(A \lambda, \Omega_{R}\left(x_{0}\right)\right)\right| \\
\leq & c \epsilon^{2} \sum_{i \geq 1}\left|\Omega_{r_{i}}\left(y_{i}\right) \cap E\left(\frac{\lambda}{4}, \Omega_{2 R}\left(x_{0}\right)\right)\right|+c \epsilon^{2} \frac{\eta}{(\zeta \lambda)^{\eta}} \sum_{i \geq 1} \int_{\zeta \lambda}^{\infty} \mu^{\eta}\left|\left\{x \in \Omega_{r_{i}}\left(y_{i}\right):|\mathbf{F}|^{\frac{2 p(x)}{P^{-}}}>\mu\right\}\right| \frac{d \mu}{\mu} \\
\leq & c \epsilon^{2}\left|E\left(\frac{\lambda}{4}, \Omega_{2 R}\left(x_{0}\right)\right)\right|+c \epsilon^{2} \frac{\eta}{(\zeta \lambda)^{\eta}} \int_{\zeta \lambda}^{\infty} \mu^{\eta}\left|\left\{x \in \Omega_{2 R}\left(x_{0}\right):|\mathbf{F}|^{\frac{2 p(x)}{P^{-}}}>\mu\right\}\right| \frac{d \mu}{\mu} .
\end{aligned}
$$

Step 4. For the case $0<q<\infty$, thanks to (2.3) in Proposition 2.2 we have

$$
\begin{gathered}
\left\||D u|^{p(x)}\right\|_{L(t, q)\left(\Omega_{R}\left(x_{0}\right)\right)}^{q}=\left\||D u|^{\frac{2 p(x)}{p^{-}}}\right\|_{L\left(\frac{p^{-}}{2} q\right.}^{\left.\frac{t p^{-}}{2}, \frac{q p^{-}}{2}\right)\left(\Omega_{R}\left(x_{0}\right)\right)} \\
=\frac{t p^{-}}{2} \int_{0}^{\infty}\left(\left.\mu^{\frac{t p^{-}}{2}}\left|x \in \Omega_{R}\left(x_{0}\right):\right| D u\right|^{\frac{2 p(x)}{p^{-}}}>\mu \mid\right)^{\frac{q}{t}} \frac{d \mu}{\mu} .
\end{gathered}
$$

For $t>1$ and $q<\infty$, we multiply inequality (3.27) by $\left(\frac{t p^{-}}{2}\right)^{\frac{t}{q}}(A \lambda)^{\frac{t p^{-}}{2}}$, and raise both sides to the power $\frac{q}{t}$ and integrate with respect to the measure $\frac{d \lambda}{A \lambda}$ over $M \lambda_{0}$ due to $A \geq 1$ being a constant depending only on $n, \gamma_{1}, \gamma_{2}, v, \Lambda$ and $\zeta$ depends on $\delta$. Then we get that

$$
\begin{aligned}
& \frac{t p^{-}}{2} \int_{M \lambda_{0}}^{\infty}\left((A \lambda)^{\frac{t p^{-}}{2}}\left|\left\{x \in \Omega_{R}\left(x_{0}\right):|D u|^{\frac{2 p(x)}{p^{-}}}>A \lambda\right\}\right|\right)^{\frac{q}{t}} \frac{d \lambda}{A \lambda} \\
\leq & c \epsilon^{\frac{2 q}{t}} \frac{t p^{-}}{2} \int_{0}^{\infty}\left(\lambda^{\frac{t p^{-}}{2}}\left|\left\{x \in \Omega_{2 R}\left(x_{0}\right):|D u|^{\frac{2 p(x)}{p^{-}}}>\frac{\lambda}{4}\right\}\right|\right)^{\frac{q}{t}} \frac{d \lambda}{\lambda} \\
& +c \epsilon^{\frac{2 q}{t}} \frac{t p^{-}}{2} \int_{0}^{\infty} \lambda^{q\left(\frac{p^{-}}{2}-\frac{\eta}{t}\right)}\left(\int_{\zeta \lambda}^{\infty} \mu^{\eta}\left|\left\{x \in \Omega_{2 R}\left(x_{0}\right):|\mathbf{F}|^{\frac{2 p(x)}{p^{-}}}>\mu\right\}\right| \frac{d \mu}{\mu}\right)^{\frac{q}{t}} \frac{d \lambda}{\lambda} \\
:= & c \epsilon^{\frac{2 q}{t}}\left(I_{1}+I_{2}\right),
\end{aligned}
$$

where $c$ depends on $n, \gamma_{1}, \gamma_{2}, v, \Lambda, q, t, \omega(\cdot)$. A simple change of variable yields

$$
I_{1}=c(q)\left\||D u|^{p(x)}\right\|_{L(t, q)\left(\Omega_{2 R}\left(x_{0}\right)\right)}^{q} .
$$

For the estimate $I_{2}$, we part it in two cases.

Case 1. If $q \geq t$, noticing that (2.4) is satisfied since $|\mathbf{F}|^{\frac{2 p(x)}{p^{-}}} \in L^{\eta}\left(\Omega_{2 R}\left(x_{0}\right)\right)$. By making the change of variables $\bar{\lambda}=\zeta \lambda$, and $\zeta=\frac{\delta}{4}$, then we use Lemma 2.3 with

$$
f(\mu)=\mu^{\eta-1}\left|\left\{x \in \Omega_{2 R}\left(x_{0}\right):|\mathbf{F}|^{\frac{2 p(x)}{P^{-}}}>\mu\right\}\right|
$$


and $\alpha=\frac{q}{t} \geq 1, r=q\left(\frac{p^{-}}{2}-\frac{\eta}{t}\right)>0$ to infer

$$
\begin{aligned}
I_{2} & =c\left(\gamma_{1}, \gamma_{2}, q, t\right) \frac{t p^{-}}{2} \int_{0}^{\infty}(\bar{\lambda})^{q\left(\frac{p^{-}}{2}-\frac{\eta}{t}\right)}\left(\int_{\bar{\lambda}}^{\infty} \mu^{\eta}\left|\left\{x \in \Omega_{2 R}\left(x_{0}\right):|\mathbf{F}|^{\frac{2 p(x)}{p^{-}}}>\mu\right\}\right| \frac{d \mu}{\mu}\right)^{\frac{q}{t}} \frac{d \bar{\lambda}}{\bar{\lambda}} \\
& \leq c \frac{t p^{-}}{2} \int_{0}^{\infty} \bar{\lambda}^{\frac{q p^{-}}{2}}\left|\left\{x \in \Omega_{2 R}\left(x_{0}\right):|\mathbf{F}|^{\frac{2 p(x)}{p^{-}}}>\bar{\lambda}\right\}\right|^{\frac{q}{t}} \frac{d \bar{\lambda}}{\bar{\lambda}} \\
& =c\left\||\mathbf{F}|^{p(x)}\right\|_{L(t, q)\left(\Omega_{2 R}\left(x_{0}\right)\right)}^{q},
\end{aligned}
$$

where $c=c\left(\gamma_{1}, \gamma_{2}, q, t\right)$.

Case 2. If $0<q<t$, we use Lemma 2.4 with $h(\mu)=\left|\left\{x \in \Omega_{2 R}\left(x_{0}\right):|\mathbf{F}|^{\frac{2 p(x)}{p^{-}}}>\mu\right\}\right|^{\frac{q}{t}}, r=\frac{\eta q}{t}, \alpha_{1}=1<\frac{t}{q}=\alpha_{2}$ and $\varepsilon=1$, then it yields

$$
\begin{aligned}
& \left(\int_{\lambda}^{\infty} \mu^{\eta}\left|\left\{x \in \Omega_{2 R}\left(x_{0}\right):|\mathbf{F}|^{\frac{2 p(x)}{p^{-}}}>\mu\right\}\right| \frac{d \mu}{\mu}\right)^{\frac{q}{t}} \\
& \leq \lambda^{\frac{\eta q}{t}}\left|\left\{x \in \Omega_{2 R}\left(x_{0}\right):|\mathbf{F}|^{\frac{2 p(x)}{p^{-}}}>\lambda\right\}\right|^{\frac{q}{t}}+c \int_{\lambda}^{\infty} \mu^{\frac{\eta q}{t}}\left|\left\{x \in \Omega_{2 R}\left(x_{0}\right):|\mathbf{F}|^{\frac{2 p(x)}{p^{-}}}>\mu\right\}\right|^{\frac{q}{t}} \frac{d \mu}{\mu} .
\end{aligned}
$$

Therefore, after changing variable $\zeta \lambda \rightarrow \lambda$ again and Fubini's theorem, we have

$$
\begin{aligned}
I_{2} \leq & c \frac{t p^{-}}{2} \int_{0}^{\infty} \lambda^{q\left(\frac{p^{-}}{2}-\frac{\eta}{t}\right)}\left(\lambda^{\frac{\eta q}{t}}\left|\left\{x \in \Omega_{2 R}\left(x_{0}\right):|\mathbf{F}|^{\frac{2 p(x)}{p^{-}}}>\lambda\right\}\right|^{\frac{q}{t}}\right. \\
& \left.+\int_{\lambda}^{\infty} \mu^{\frac{\eta q}{t}-1}\left|\left\{x \in \Omega_{2 R}\left(x_{0}\right):|\mathbf{F}|^{\frac{2 p(x)}{p^{-}}}>\mu\right\}\right|^{\frac{q}{t}} d \mu\right) \frac{d \lambda}{\lambda} \\
\leq & c\left\||\mathbf{F}|^{p(x)}\right\|_{L(t, q)\left(\Omega_{2 R}\left(x_{0}\right)\right)}^{q}+c \frac{t p^{-}}{2} \int_{0}^{\infty} \lambda^{q\left(\frac{p^{-}}{2}-\frac{\eta}{t}\right)}\left(\int_{\lambda}^{\infty} \mu^{\frac{\eta q}{t}-1}\left|\left\{x \in \Omega_{2 R}\left(x_{0}\right):|\mathbf{F}|^{\frac{2 p(x)}{p^{-}}}>\mu\right\}\right|^{\frac{q}{t}} d \mu\right) \frac{d \lambda}{\lambda} \\
\leq & c\left\||\mathbf{F}|^{p(x)}\right\|_{L(t, q)\left(\Omega_{2 R}\left(x_{0}\right)\right)}^{q},
\end{aligned}
$$

where $c=c\left(\gamma_{1}, \gamma_{2}, q, t\right)$.

Let us insert the estimates of $I_{1}, I_{2}$ into (3.29), for all $t>1$ by simple manipulations it leads to that

$$
\begin{aligned}
& \left\||D u|^{p(x)}\right\|_{L(t, q)\left(\Omega_{R}\left(x_{0}\right)\right)} \\
\leq & c\left(\frac{t p^{-}}{2} \int_{M \lambda_{0}}^{\infty}\left((A \lambda)^{\frac{t p^{-}}{2}}\left|\left\{x \in \Omega_{R}\left(x_{0}\right):|D u|^{\frac{2 p(x)}{p^{-}}}>A \lambda\right\}\right|\right)^{\frac{q}{t}} \frac{d(A \lambda)}{A \lambda}\right)^{\frac{1}{q}} \\
& +c\left(\frac{t p^{-}}{2} \int_{0}^{M \lambda_{0}}\left((A \lambda)^{\frac{t p^{-}}{2}}\left|\left\{x \in \Omega_{R}\left(x_{0}\right):|D u|^{\frac{2 p(x)}{p^{-}}}>A \lambda\right\}\right|\right)^{\frac{q}{t}} \frac{d(A \lambda)}{A \lambda}\right)^{\frac{1}{q}} \\
\leq & c\left(\frac{t p^{-}}{2} \int_{0}^{M \lambda_{0}}\left((A \lambda)^{\frac{t p^{-}}{2}}\left|\left\{x \in \Omega_{R}\left(x_{0}\right):|D u|^{\frac{2 p(x)}{p^{-}}}>A \lambda\right\}\right|\right)^{\frac{q}{t}} \frac{d(A \lambda)}{A \lambda}\right)^{\frac{1}{q}} \\
& +\bar{c} \epsilon^{\frac{2}{t}}\left(\left\||D u|^{p(x)}\right\|_{L(t, q)\left(\Omega_{2 R}\left(x_{0}\right)\right)}+\left\||\mathbf{F}|^{p(x)}\right\|_{L(t, q)\left(\Omega_{2 R}\left(x_{0}\right)\right)}\right) \\
\leq & c \lambda_{0}^{\frac{p^{-}}{2}}\left|\Omega_{2 R}\left(x_{0}\right)\right|^{\frac{1}{t}}+\bar{c} \epsilon^{\frac{2}{t}}\left(\left\||D u|^{p(x)}\right\|_{L(t, q)\left(\Omega_{2 R}\left(x_{0}\right)\right)}+\left\||\mathbf{F}|^{p(x)}\right\|_{L(t, q)\left(\Omega_{2 R}\left(x_{0}\right)\right)}\right),
\end{aligned}
$$

where $\bar{c}=\bar{c}\left(n, \gamma_{1}, \gamma_{2}, \nu, \Lambda, q, t, \omega(\cdot)\right)$. It suffices to choose first $\epsilon>0$ small enough that $\bar{c} \epsilon^{\frac{2}{t}} \leq \frac{1}{2}$. Once the selection of $\epsilon$ is made, we can find corresponding constants $\delta=\delta\left(\epsilon, \gamma_{1}, \gamma_{2}\right)$. Then we deduce

$$
\left\||D u|^{p(x)}\right\|_{L(t, q)\left(\Omega_{R}\left(x_{0}\right)\right)} \leq c \lambda_{0}^{\frac{p^{-}}{2}}\left|\Omega_{2 R}\left(x_{0}\right)\right|^{\frac{1}{t}}+\frac{1}{2}\left\||D u|^{p(x)}\right\|_{L(t, q)\left(\Omega_{2 R}\left(x_{0}\right)\right)}+c\left\||\mathbf{F}|^{p(x)}\right\|_{L(t, q)\left(\Omega_{2 R}\left(x_{0}\right)\right)} .
$$


We now claim that $\left\||D u|^{p(x)}\right\|_{L(t, q)\left(\Omega_{2 R}\left(x_{0}\right)\right)}<\infty$, which will be proved in the next step. By a standard iteration argument lemma 2.5 we get an estimate similar to (1.12) in the case $t>1$ and $0<q<\infty$.

Step 5. In this step we focus on proving the above claim: $\left\||D u|^{p(x)}\right\|_{L(t, q)\left(\Omega_{2 R}\left(x_{0}\right)\right)}<\infty$. To this end, we first show how to refine the estimate of $|D u|^{p(x)}$ in the scale of Lorentz spaces. Consider the truncated function:

$$
\left.\left.|| D u\right|^{p(x)}\right|_{k}=|D u|^{p(x)} \wedge k \text { for } x \in \Omega \text { and } k \in \mathbb{N} \cap\left[M \lambda_{0}, \infty\right) .
$$

Note that for $E_{k}\left(\lambda, \Omega_{\rho}\left(x_{0}\right)\right)=\left\{x \in \Omega_{\rho}\left(x_{0}\right):\left.\left.|| D u\right|^{p(x)}\right|_{k}>\lambda\right\}$ in line with (3.27), we have

$$
E_{k}\left(A \lambda, \Omega_{R}\left(x_{0}\right)\right) \leq c \epsilon^{2}\left|E_{k}\left(\frac{\lambda}{4}, \Omega_{2 R}\left(x_{0}\right)\right)\right|+c \epsilon^{2} \frac{\eta}{(\zeta \lambda)^{\eta}} \int_{\zeta \lambda}^{\infty} \mu^{\eta}\left|\left\{x \in \Omega_{2 R}\left(x_{0}\right):|\mathbf{F}|^{\frac{2 p(x)}{p^{-}}}>\mu\right\}\right| \frac{d \mu}{\mu}
$$

for $k \in \mathbb{N} \cap\left[M \lambda_{0}, \infty\right)$. Indeed, for $k \leq A \lambda$ we have $E_{k}\left(A \lambda, \Omega_{R}\left(x_{0}\right)\right)=\emptyset$, which implies that the above estimate holds trivially. For $k>A \lambda$, it is also valid due to $E_{k}\left(A \lambda, \Omega_{R}\left(x_{0}\right)\right)=E\left(A \lambda, \Omega_{R}\left(x_{0}\right)\right)=\left\{x \in \Omega_{R}\left(x_{0}\right),|D u|^{p(x)}>\right.$ $A \lambda\}$ and $E_{k}\left(\frac{\lambda}{4}, \Omega_{2 R}\left(x_{0}\right)\right)=E\left(\frac{\lambda}{4}, \Omega_{2 R}\left(x_{0}\right)\right)$. Working exactly as in the above argument, we get that (3.31) holds true with $\left.\left.|| D u\right|^{p(x)}\right|_{k}$ in place of $|D u|^{p(x)}$. Now let $B_{0}=0, L=c \lambda_{0}^{\frac{p^{-}}{2}}\left|\Omega_{2 R}\left(x_{0}\right)\right|^{\frac{1}{t}}+c\left\||\mathbf{F}|^{p(x)}\right\|_{L(t, q)\left(\Omega_{2 R}\left(x_{0}\right)\right)}$ and $\varphi(\rho)=\left\|\left.\left.|| D u\right|^{p(x)}\right|_{k}\right\|_{L(t, q)\left(\Omega_{\rho}\left(x_{0}\right)\right)}$. We use a well-known iteration argument of Lemma 2.5 due to

$$
\left\|\left.\left.|| D u\right|^{p(x)}\right|_{k}\right\|_{L(t, q)\left(\Omega_{R}\left(x_{0}\right)\right)}<\infty,
$$

and get

$$
\left\|\left.\left.|| D u\right|^{p(x)}\right|_{k}\right\|_{L(t, q)\left(\Omega_{R}\left(x_{0}\right)\right)} \leq c \lambda_{0}^{\frac{p^{-}}{2}}\left|\Omega_{R}\left(x_{0}\right)\right|^{\frac{1}{t}}+c\left\||\mathbf{F}|^{p(x)}\right\|_{L(t, q)\left(\Omega_{2 R}\left(x_{0}\right)\right)} .
$$

In what follows, we make use of a standard finite covering argument to realize our global estimate. Note that $\Omega$ is bounded domain in $\mathbb{R}^{n}$ and $x_{0}$ is any fixed point of $\Omega$. Then there exist $N \in \mathbb{N}$ and $x_{j} \in \Omega$ for $j=1,2, \cdots, N$, where one replaces the point $x_{0}$ by each $x_{j}$, such that

$$
\bar{\Omega} \subset \cup_{j=1}^{N} \Omega_{R}\left(x_{j}\right) .
$$

Therefore, by (3.32) we have

$$
\begin{aligned}
& \left\|\left.\left.|| D u\right|^{p(x)}\right|_{k}\right\|_{L(t, q)(\Omega)} \\
\leq & \left.\left.\sum_{j=1}^{N}|||| D u\right|^{p(x)}\right|_{k} \|_{L(t, q)\left(\Omega_{R}\left(x_{j}\right)\right)} \\
\leq & c \sum_{j=1}^{N}\left(\lambda_{0}^{\frac{p^{-}}{2}}\left|\Omega_{R}\left(x_{j}\right)\right|^{\frac{1}{t}}+\left\||\mathbf{F}|^{p(x)}\right\|_{L(t, q)\left(\Omega_{2 R}\left(x_{j}\right)\right)}\right) \\
\leq & c \sum_{j=1}^{N}\left(\lambda_{0}^{\frac{p^{-}}{2}}\left|\Omega_{R}\left(x_{j}\right)\right|^{\frac{1}{t}}+\left\||\mathbf{F}|^{p(x)}\right\|_{L(t, q)\left(\Omega_{2 R}\left(x_{j}\right)\right)}\right) .
\end{aligned}
$$

Recalling the definition of $\lambda_{0}$, we get

$$
\begin{aligned}
& \left\|\left.|D u|^{p(x)}\right|_{k}\right\|_{L(t, q)(\Omega)} \\
\leq & c \sum_{j=1}^{N}\left|\Omega_{R}\left(x_{j}\right)\right|^{\frac{1}{t}}\left(f_{\Omega_{2 R}\left(x_{j}\right)}|D u|^{\frac{2 p(x)}{p^{-}}} d x+\left(f_{\Omega_{2 R}\left(x_{j}\right)}\left(|\mathbf{F}|^{\frac{2 p(x)}{p^{-}}}+1\right)^{\eta} d x\right)^{\frac{1}{\eta}}\right)^{\frac{p^{-}}{2}}+c \sum_{j=1}^{N}\left\||\mathbf{F}|^{p(x)}\right\|_{L(t, q)\left(\Omega_{2 R}\left(x_{j}\right)\right)}
\end{aligned}
$$




$$
\leq c \sum_{j=1}^{N}\left|\Omega_{R}\left(x_{j}\right)\right|^{\frac{1}{t}}\left(f_{\Omega_{2 R}\left(x_{j}\right)}|D u|^{\frac{2 p(x)}{p^{-}}} d x+\left(f_{\Omega_{2 R}\left(x_{j}\right)}\left(|\mathbf{F}|^{\frac{2 p(x)}{p^{-}}}+1\right)^{\eta} d x\right)^{\frac{1}{\eta}}\right)^{\frac{p^{-}}{2}}+c N\left\||\mathbf{F}|^{p(x)}\right\|_{L(t, q)(\Omega)} .
$$

Noticing that

$$
\frac{2 p^{+}}{p^{-}}=2\left(1+\frac{p^{+}-p^{-}}{p^{-}}\right) \leq 2\left(1+\frac{\omega(2 R)}{\gamma_{1}}\right) \leq 2(1+\sigma),
$$

where $\sigma$ is the same as Lemma 2.7. Then, it yields

$$
\begin{aligned}
& f_{\Omega_{2 R}\left(x_{j}\right)}|D u|^{\frac{2 p(x)}{p^{-}}} d x \\
\leq & f_{\Omega_{2 R}\left(x_{j}\right)}|D u|^{\frac{2 p^{+}}{p^{-}}} d x+1 \\
\leq & c\left(f_{\Omega_{4 R}\left(x_{j}\right)}|D u|^{2} d x\right)^{\frac{p^{+}}{p^{-}}}+f_{\Omega_{4 R}\left(x_{j}\right)}|\mathbf{F}|^{\frac{2 p^{+}}{p^{-}}} d x+1,
\end{aligned}
$$

where we have employed the reverse Höder inequality Lemma 2.7 in the last inequality. By using Höder inequality, we have

$$
\begin{aligned}
& \left(f_{\Omega_{4 R}\left(x_{j}\right)}|D u|^{2} d x\right)^{\frac{p^{+}}{p^{-}}} \\
\leq & \left(\frac{1}{\mid \Omega_{4 R}\left(x_{j}\right)}\right)^{\frac{p^{+}}{p^{-}}}\left(\int_{\Omega}|D u|^{2} d x\right)^{\frac{p^{+}}{p^{-}}} \\
\leq & c\left(\frac{1}{\left|\Omega_{4 R}\left(x_{j}\right)\right|}\right)^{\frac{p^{+}}{p^{-}}}\left(\int_{\Omega}|\mathbf{F}|^{2} d x\right)^{\frac{p^{+}}{p^{-}}} \\
\leq & c\left(\frac{1}{\left|\Omega_{4 R}\left(x_{j}\right)\right|}\right)^{\frac{p^{+}}{p^{-}}}|\Omega|^{1-\frac{p^{-}}{p^{+}}} \int_{\Omega}|\mathbf{F}|^{\frac{2 p^{+}}{p^{-}}} d x \\
\leq & c\left(\frac{1}{\left|\Omega_{4 R}\left(x_{j}\right)\right|}\right)^{\frac{p^{+}}{p^{-}}} \int_{\Omega}\left(|\mathbf{F}|^{\frac{2 p(x)}{p^{-}}{\frac{p}{p^{+}}}^{p^{-}}}+1\right) d x .
\end{aligned}
$$

Combining (3.36), (3.34) and (3.35), we get that

$$
\begin{aligned}
& \left\|\left.\left.|| D u\right|^{p(x)}\right|_{k}\right\|_{L(t, q)(\Omega)} \\
\leq & c \sum_{j=1}^{N}\left|\Omega_{R}\left(x_{j}\right)\right|^{\frac{1}{t}}\left(\left(\frac{1}{\left|\Omega_{4 R}\left(x_{j}\right)\right|}\right)^{\frac{p^{+}}{p^{-}}} \int_{\Omega}\left(|\mathbf{F}|^{\frac{2 p(x)}{p^{-}} \frac{p^{+}}{p^{-}}}+1\right) d x+\left(f_{\Omega_{2 R}\left(x_{j}\right)}\left(|\mathbf{F}|^{\frac{2 p(x)}{p^{-}}}+1\right)^{\eta} d x\right)^{\frac{1}{\eta}}\right)^{\frac{p^{-}}{2}}+c N\left\|\left.\mathbf{F}\right|^{p(x)}\right\|_{L(t, q)(\Omega)} \\
\leq & c \sum_{j=1}^{N}\left|\Omega_{R}\left(x_{j}\right)\right|^{\frac{1}{t}}\left(\left(\frac{1}{\left|\Omega_{4 R}\left(x_{j}\right)\right|}\right)^{\frac{p^{+}}{p^{-}}} \int_{\Omega}\left(|\mathbf{F}|^{\frac{2 p(x)}{p^{-}} \frac{p^{+}}{p^{-}}}+1\right) d x+\left(\frac{1}{\left|\Omega_{2 R}\left(x_{j}\right)\right|}\right)^{\frac{1}{\eta}}\left(\int_{\Omega}\left(|\mathbf{F}|^{\frac{2 p(x)}{p^{-}}}+1\right)^{\eta} d x\right)^{\frac{1}{\eta}}\right)^{\frac{p^{-}}{2}} \\
& +c N\left\||\mathbf{F}|^{p(x)}\right\|_{L(t, q)(\Omega)} .
\end{aligned}
$$

Making use of a standard Hardy's inequality in Marcinkiewicz-spaces (cf. Lemma 2.3 in [24]) and Lemma 2.4 , it yields

$$
\int_{\Omega}|\mathbf{F}|^{\frac{2 p(x)}{p^{-}} \frac{p^{+}}{p^{-}}} d x
$$




$$
\begin{aligned}
& \leq \frac{t\left(p^{-}\right)^{2}}{t\left(p^{-}\right)^{2}-2 p^{+}}|\Omega|^{1-\frac{2 p^{+}}{t\left(p^{-}\right)^{2}}}\left\||\mathbf{F}|^{\frac{2 p(x)}{p^{-}}}\right\|_{\mathcal{M}^{\frac{p^{+}}{p^{-}}}}^{\frac{t p^{-}}{p^{-}}(\Omega)} \\
& =\frac{t\left(p^{-}\right)^{2}}{t\left(p^{-}\right)^{2}-2 p^{+}}|\Omega|^{1-\frac{2 p^{+}}{t\left(p^{-}\right)^{2}}}\left(\sup _{h>0}\left(h^{\frac{t p^{-}}{2}}\left|\left\{x \in \Omega:|\mathbf{F}|^{\frac{2 p(x)}{p^{-}}}>h\right\}\right|\right)^{\frac{2}{t p^{-}}}\right)^{\frac{p^{+}}{p^{-}}} \\
& \leq c|\Omega|^{1-\frac{2 p^{+}}{t\left(p^{-}\right)^{2}}}\left\||\mathbf{F}|^{\frac{2 p(x)}{p^{-}}}\right\|_{L\left(\frac{p^{+}}{p^{-}}\right.}^{\left.\frac{t p^{-}}{2}, \frac{q p^{-}}{2}\right)(\Omega)} \\
& \leq c|\Omega|^{1-\frac{2 p^{+}}{t\left(p^{-}\right)^{2}}}\left\||\mathbf{F}|^{p(x)}\right\|_{L(t, q)(\Omega)}^{\frac{2 p^{+}}{\left(p^{-}\right)^{2}}} .
\end{aligned}
$$

Similarly, we derive

$$
\begin{aligned}
& \left(\int_{\Omega}|\mathbf{F}|^{\frac{2 p(x)}{p^{-}} \eta} d x\right)^{\frac{1}{\eta}} \\
\leq & c\left(\gamma_{1}, \gamma_{2}, q, t\right)|\Omega|^{\frac{1}{\eta}-\frac{2}{t p^{-}}}\left\||\mathbf{F}|^{\frac{2 p(x)}{p^{-}}}\right\|_{L\left(\frac{t p^{-}}{2}, \frac{q p^{-}}{2}\right)(\Omega)} \\
\leq & c\left(\gamma_{1}, \gamma_{2}, q, t\right)|\Omega|^{\frac{1}{\eta}-\frac{2}{t p^{-}}}\left\||\mathbf{F}|^{p(x)}\right\|_{L(t, q)(\Omega)}^{\frac{2}{p^{-}}} .
\end{aligned}
$$

In the case of $q<\infty$, from (3.36) we then infer

$$
\begin{aligned}
& \left\|\left.|D u|^{p(x)}\right|_{k}\right\|_{L(t, q)(\Omega)} \\
\leq & c \sum_{j=1}^{N}\left(\left(\frac{|\Omega|}{\left|\Omega_{2 R}\left(x_{j}\right)\right|}\right)^{\frac{p^{+}}{2}-\frac{1}{t}}\left(\left\||\mathbf{F}|^{p(x)}\right\|_{L(t, q)(\Omega)}^{\frac{p^{+}}{p^{-}}}+1\right)+\left(\frac{|\Omega|}{\left|\Omega_{2 R}\left(x_{j}\right)\right|}\right)^{\frac{p^{-}}{2 \eta}-\frac{1}{t}}\left(\left\||\mathbf{F}|^{p(x)}\right\|_{L(t, q)(\Omega)}+1\right)\right) \\
\leq & c \sum_{j=1}^{N}\left(\left(\frac{|\Omega|}{\left|Q_{2 R}\left(x_{j}\right)\right|} \frac{\left|Q_{2 R}\left(x_{j}\right)\right|}{\left|\Omega_{2 R}\left(x_{j}\right)\right|}\right)^{\frac{p^{+}}{2}-\frac{1}{t}}\left(\left\||\mathbf{F}|^{p(x)}\right\|_{L(t, q)(\Omega)}^{\frac{p^{+}}{p^{-}}}+1\right)+\left(\frac{|\Omega|}{\left|Q_{2 R}\left(x_{j}\right)\right|} \frac{\left|Q_{2 R}\left(x_{j}\right)\right|}{\left|\Omega_{2 R}\left(x_{j}\right)\right|}\right)^{\frac{p^{-}}{2 \eta}-\frac{1}{t}}\left(\left\||\mathbf{F}|^{p(x)}\right\|_{L(t, q)(\Omega)}+1\right)\right) \\
\leq & c \sum_{j=1}^{N}\left(\left(\frac{|\Omega|}{\left|Q_{2 R}\left(x_{j}\right)\right|}\left(\frac{2 \sqrt{2}}{1-\delta}\right)^{n}\right)^{\frac{p^{+}}{2}-\frac{1}{t}}\left(\left\||\mathbf{F}|^{p(x)}\right\|_{L(t, q)(\Omega)}^{\frac{p^{+}}{p^{-}}}+1\right)+\left(\frac{|\Omega|}{\left|Q_{2 R}\left(x_{j}\right)\right|}\left(\frac{2 \sqrt{2}}{1-\delta}\right)^{n}\right)^{\frac{p^{-}}{2 \eta}-\frac{1}{t}}\left(\left\||\mathbf{F}|^{p(x)}\right\|_{L(t, q)(\Omega)}+1\right)\right) \\
\leq & c N\left(\left\||\mathbf{F}|^{p(x)}\right\|_{L(t, q)(\Omega)}+1\right)^{\frac{p^{+}}{p^{-}}} \\
\leq & c N\left(\left\||\mathbf{F}|^{p(x)}\right\|_{L(t, q)(\Omega)}+1\right)^{\frac{\gamma_{2}}{\gamma_{1}}}
\end{aligned}
$$

Then, let us take $k \rightarrow \infty$, by the lower semi-continuity of Lorentz quasi-norm we have

$$
\left\||D u|^{p(x)}\right\|_{L(t, q)(\Omega)} \leq c N\left(\left\||\mathbf{F}|^{p(x)}\right\|_{L(t, q)(\Omega)}+1\right)^{\frac{\gamma_{2}}{\gamma_{1}}}
$$

where $c$ depends only on $n, \gamma_{1}, \gamma_{2}, \nu, \Lambda, t, q, \omega(\cdot), R_{0},|\Omega|$.

Step 6. For the case of $q=\infty$, we come back to the second inequality in (3.6) and split it in two parts with a small $\iota>0$ determined later as follows:

$$
\left(\frac{\lambda}{2}\right)^{\eta} \leq \frac{1}{\delta^{\eta}} f_{\Omega_{r_{y}}(y)}|\mathbf{F}|^{\frac{2 p(x)}{p^{-}} \eta} d x \leq \frac{(\iota \lambda)^{\eta}}{\delta^{\eta}}+\frac{1}{\delta^{\eta}\left|\Omega_{r_{y}}(y)\right|} \int_{\left\{x \in \Omega_{r y}(y):|\mathbf{F}|^{\left.\frac{2 p(x)}{p^{-}}>\lambda \lambda\right\}}\right.}|\mathbf{F}|^{\frac{2 p(x)}{p^{-}} \eta} d x .
$$


Set

$$
\left\{\begin{array}{l}
G\left(\iota \lambda, \Omega_{r_{y}}(y)\right)=\left\{x \in \Omega_{r_{y}}(y):|\mathbf{F}|^{\frac{2 p(x)}{p^{-}}}>\iota \lambda\right\}, \\
G\left(\mu, \Omega_{r_{y}}(y)\right)=\left\{x \in \Omega_{r_{y}}(y):|\mathbf{F}|^{\frac{2 p(x)}{p^{-}}}>\mu\right\}
\end{array}\right.
$$

then, similar to (3.37) by using the Hölder inequality we get

$$
\begin{aligned}
& \left(\frac{\lambda}{2}\right)^{\eta}-\left(\frac{\iota \lambda}{\delta}\right)^{\eta} \\
\leq & \frac{1}{\delta^{\eta}\left|\Omega_{r_{y}}(y)\right|} \int_{\left\{x \in \Omega_{r_{y}}(y):|\mathbf{F}|^{\left.\frac{2 p(x)}{p^{-}}>\iota \lambda\right\}}\right.}|\mathbf{F}|^{\frac{2 p(x)}{p^{-}} \eta} d x \\
\leq & \frac{t}{(t-\eta) \delta^{\eta}} \frac{\left|G\left(\iota \lambda, \Omega_{r_{y}}(y)\right)\right|^{1-\frac{\eta}{t}}}{\left|\Omega_{r_{y}}(y)\right|} \sup _{\mu>0} \mu^{\eta}\left|\left\{x \in G\left(\iota \lambda \Omega_{r_{y}}(y)\right):|\mathbf{F}|^{\frac{2 p(x)}{p^{-}} \eta} \geq \mu\right\}\right|^{\frac{\eta}{t}} \\
\leq & \frac{t\left|G\left(\iota \lambda, \Omega_{r_{y}}(y)\right)\right|^{1-\frac{\eta}{t}}}{(t-\eta) \delta^{\eta}\left|\Omega_{r_{y}}(y)\right|}\left((\iota \lambda)^{\eta}\left|G\left(\iota \lambda, \Omega_{r_{y}}(y)\right)\right|^{\frac{\eta}{t}}+\sup _{\mu>\iota \lambda} \mu^{\eta}\left|G\left(\mu, \Omega_{r_{y}}(y)\right)\right|^{\frac{\eta}{t}}\right) \\
= & \frac{t}{(t-\eta) \delta^{\eta}}\left((\iota \lambda)^{\eta}+\frac{\left|G\left(\iota \lambda, \Omega_{r_{y}}(y)\right)\right|^{1-\frac{\eta}{t}}}{\left|\Omega_{r_{y}}(y)\right|} \sup _{\mu>\iota \lambda} \mu^{\eta}\left|G\left(\mu, \Omega_{r_{y}}(y)\right)\right|^{\frac{\eta}{t}}\right) .
\end{aligned}
$$

Now we choose $\iota>0$ sufficiently small with

$$
\left(\frac{\lambda}{2}\right)^{\eta}-\left(\frac{\iota \lambda}{\delta}\right)^{\eta}-\frac{t}{t-\eta}\left(\frac{\iota \lambda}{\delta}\right)^{\eta}=\left(\frac{\lambda}{2}\right)^{\eta}-\left(\frac{\iota \lambda}{\delta}\right)^{\eta}\left(1+\frac{t}{t-\eta}\right) \geq\left(\frac{\lambda}{4}\right)^{\eta}
$$

which implies that there exists a positive constant $c(t)$ depending only on $t$ such that

$$
\iota \leq c(t) \delta
$$

Therefore

$$
\begin{aligned}
\left|\Omega_{r_{y}}(y)\right| & \leq \frac{c t}{t-\eta} \frac{\left|G\left(\iota \lambda, \Omega_{r_{y}}(y)\right)\right|^{1-\frac{\eta}{t}}}{(\iota \lambda)^{\eta}}\left(\sup _{\mu>\iota \lambda} \mu^{t}\left|G\left(\mu, \Omega_{r_{y}}(y)\right)\right|\right)^{\frac{\eta}{t}} \\
& \leq \frac{c t(\iota \lambda)^{-t}}{t-\eta}\left((\iota \lambda)^{t}\left|G\left(\iota \lambda, \Omega_{r_{y}}(y)\right)\right|\right)^{1-\frac{\eta}{t}}\left(\sup _{\mu>\iota \lambda} \mu^{t}\left|G\left(\mu, \Omega_{r_{y}}(y)\right)\right|\right)^{\frac{\eta}{t}} \\
& \leq \frac{c t(\iota \lambda)^{-t}}{t-\eta} \sup _{\mu>\iota \lambda} \mu^{t}\left|G\left(\mu, \Omega_{r_{y}}(y)\right)\right| .
\end{aligned}
$$

Now we put the estimates of the two terms in (3.6) together into (3.26), which means that we insert the formulas (3.7) and (3.38) in (3.26) to get

$$
E\left(A \lambda, \Omega_{R}\left(x_{0}\right)\right) \leq c \epsilon\left|E\left(\frac{\lambda}{4}, \Omega_{2 R}\left(x_{0}\right)\right)\right|+c \epsilon(\iota \lambda)^{-t} \sup _{\mu>\imath \lambda} \mu^{t}\left|G\left(\mu, \Omega_{2 R}\left(x_{0}\right)\right)\right| .
$$

Multiplying (3.39) by $(A \lambda)^{\frac{t p^{-}}{2}}$ and taking the supremum with respect to $\lambda$ over $\left(M \lambda_{0}, \infty\right)$, then we show

$$
\begin{aligned}
& \sup _{\lambda>M \lambda_{0}}(A \lambda)^{\frac{t p^{-}}{2}}\left|\left\{x \in \Omega_{R}\left(x_{0}\right):|D u|^{\frac{2 p(x)}{p^{-}}}>A \lambda\right\}\right| \\
\leq & c \epsilon^{2} A^{\frac{t p^{-}}{2}}\left(\sup _{\lambda>M \lambda_{0}} \lambda^{\frac{t p^{-}}{2}}\left|\left\{x \in \Omega_{2 R}\left(x_{0}\right):|D u|^{\frac{2 p(x)}{p^{-}}}>\frac{\lambda}{4}\right\}\right|+c\left(\gamma_{1}, \gamma_{2}, q, t\right) \sup _{\lambda>M \iota \lambda_{0}} \lambda^{\frac{t p^{-}}{2}-t}\left(\sup _{\mu>\lambda} \mu^{t}\left|G\left(\mu, \Omega_{2 R}\left(x_{0}\right)\right)\right|\right)\right)
\end{aligned}
$$




$$
\leq c \epsilon^{2}\left(\sup _{\lambda>M \lambda_{0}} \lambda^{\frac{t p^{-}}{2}}\left|\left\{x \in \Omega_{2 R}\left(x_{0}\right):|D u|^{\frac{2 p(x)}{p^{-}}}>\frac{\lambda}{4}\right\}\right|+c\left(\gamma_{1}, \gamma_{2}, q, t\right) \sup _{\lambda>M \iota \lambda_{0}}\left(\sup _{\mu>\lambda} \mu^{\frac{t p^{-}}{2}}\left|G\left(\mu, \Omega_{2 R}\left(x_{0}\right)\right)\right|\right)\right) .
$$

Note that $\sup _{\lambda>M \iota \lambda_{0}} \sup _{\mu>\lambda} \mu^{\frac{t p^{-}}{2}}\left|G\left(\mu, \Omega_{2 R}\left(x_{0}\right)\right)\right| \leq\left\||\mathbf{F}|^{p(x)}\right\|_{\mathcal{M}^{t}\left(\Omega_{2 R}\left(x_{0}\right)\right)}^{t}$, by taking $\epsilon>0$ so small to ensure that $c \epsilon^{\frac{2}{t}} \leq \frac{1}{2}$ it follows that

$$
\begin{aligned}
& \left\||D u|^{p(x)}\right\|_{\mathcal{M}^{t}\left(\Omega_{R}\left(x_{0}\right)\right)} \\
\leq & c \epsilon^{\frac{2}{t}}\left(\left\||D u|^{p(x)}\right\|_{\mathcal{M}^{t}\left(\Omega_{2 R}\left(x_{0}\right)\right)}+c\left(\gamma_{1}, \gamma_{2}, q, t\right)\left\||\mathbf{F}|^{p(x)}\right\|_{\mathcal{M}^{t}\left(\Omega_{2 R}\left(x_{0}\right)\right)}\right)+c\left|\Omega_{2 R}\left(x_{0}\right)\right|^{\frac{1}{t}} M \lambda_{0}^{\frac{p^{-}}{2}} \\
\leq & \frac{1}{2}\left\||D u|^{p(x)}\right\|_{\mathcal{M}^{t}\left(\Omega_{2 R}\left(x_{0}\right)\right)}+c\left\||\mathbf{F}|^{p(x)}\right\|_{\mathcal{M}^{t}\left(\Omega_{2 R}\left(x_{0}\right)\right)} \\
& +c\left|\Omega_{2 R}\left(x_{0}\right)\right|^{\frac{1}{t}}\left(\int_{\Omega_{2 R}\left(x_{0}\right)}|D u|^{\frac{2 p(x)}{p^{-}}} d x+\left(f_{\Omega_{2 R}\left(x_{0}\right)}\left(|\mathbf{F}|^{\frac{2 p(x)}{p^{-}}}+1\right)^{\eta} d x\right)^{\frac{1}{\eta}}\right)^{\frac{p^{-}}{2}} .
\end{aligned}
$$

Similar to the argument of Step 5, it leads to the desired result for the case $q=\infty$.

Acknowledgement The authors would like to thank the anonymous referees for their valuable comments and suggestions. This research is supported by NSFC grant 11371050 and NSFC-ERC grant 11611530539. The second author is also supported by ERCEA Advanced Grant 2014 669689-HADE, by the MINECO project MTM2014-53850-P, by Basque Government project IT-641-13 and also by the Basque Government through the BERC 2014-2017 program and by Spanish Ministry of Economy and Competitiveness MINECO: BCAM Severo Ochoa excellence accreditation SEV-2013-0323.

\section{References}

[1] E. Acerbi, G. Mingione, Gradient estimates for the $p(x)$-Laplacean system, J. Reine Angew. Math. 584 (2005) 117-148.

[2] E. Acerbi, G. Mingione, Gradient estimates for a class of parabolic systems, Duke Math. J. 136 (2) (2007) 285-320.

[3] P. Baroni, Lorentz estimates for degenerate and singular evolutionary systems, J. Differential Equations 255 (2013) 2927-2951.

[4] P. Baroni, Lorentz estimates for obstacle parabolic problems, Nonlinear Anal. 96 (2014) 167-188.

[5] P. Baroni, Marcinkiewicz estimates for degenerate parabolic equations with measure data, J. Funct. Anal. 267 (2014) 3397-3426.

[6] S. S. Byun, L. H. Wang, Elliptic equations with measurable coefficients in Reifenberg domains, Adv. Math. 225 (5) (2010) 2648-2673.

[7] S. S. Byun, Y. Cho, L. H. Wang, Calderón-Zygmund theory for nonlinear elliptic problems with irregular obstacles, J. Funct. Anal. 263 (10) (2012) 3117-3143.

[8] S. S. Byun, S. Ryu, Global weighted estimates for the gradient of solutions to nonlinear elliptic equations, Ann. Inst. H. Poincaré Anal. Non Linéaire 30 (2) (2013) 291-313.

[9] S. S. Byun, J. Ok, L. H. Wang, $W^{1, p(x)}$-Regularity for elliptic equations with measurable coefficients in nonsmooth domains, Commun. Math. Phys. 329 (2014) 937-958. 
[10] S. S. Byun, D. K. Palagachev, Weighted $L^{p}$-estimates for Elliptic Equations with Measurable Coefficients in Nonsmooth Domains, Potential Anal. 41 (2014) 51-79.

[11] S. S. Byun, Y. Kim, Elliptic equations with measurable nonlinearities innonsmooth domains, Adv. Math. 288 (2016) 152-200.

[12] L. A. Caffarelli, I. Peral, On $W^{1, p}$ estimates for elliptic equations in divergence form, Comm. Pure Appl. Math. 51 (1998) 1-21.

[13] D. V. Cruz-Uribe, A. Fiorenza, Variable Lebesgue spaces, Foundations and Harmonic Analysis, Birkhäuser, Springer-Basel, 2013.

[14] H. J. Dong, D. Kim, Elliptic equations in divergence form with partially BMO coefficients, Arch. Ration. Mech. Anal. 196 (2010) 25-70.

[15] H. J. Dong, D. Kim, On the $L_{p}$-solvability of higher order parabolic and elliptic systems with BMO coefficients, Arch. Ration. Mech. Anal. 199 (2011) 889-941.

[16] H. J. Dong, D. Kim, Global regularity of weak solutions to quasilinear elliptic and parabolic equations with controlled growth, Comm. Partial Differential Equations 36 (2011) 1750-1777.

[17] H. J. Dong, D. Kim, Parabolic and elliptic systems in divergence form with variably partially BMO coefficients, SIAM J. Math. Anal. 43 (3) (2011) 1075-1098.

[18] M. Giaquinta, Multiple integral in the calculus of variations and nonlinear elliptic systems, Princeton Univ. Press, Princeton, 1983.

[19] D. Kim, N. V. Krylov, Elliptic differential equations with coefficients measurable with respect to one variable and VMO with respect to the others, SIAM J. Math. Anal. 39 (2007) 489-506.

[20] N. V. Krylov, Parabolic and elliptic equations with VMO coefficients, Comm. Partial Differential Equations 32 (1-3) (2007) 453-475.

[21] T. Mengesha, N.C. Phuc, Global estimates for quasilinear elliptic equations on Reifenberg flat domain, Arch. Ration. Mech. Anal. 203 (2012) 189-216.

[22] N. G. Meyers, A. Elcrat, Some results on regularity for solutions of non-linear elliptic systems and quasi-regular functions, Duke Math. J. 42 (1975) 121-136.

[23] G. Mingione, Gradient potential estimates, J. Eur. Math. Soc. 13 (2011) 459-486.

[24] G. Mingione, The Calderón-Zygmund theory for elliptic problems with measure data, Ann. Sc. Norm. Super. Pisa. Cl. Sci. 6 (5) (2007) 195-261.

[25] M. V. Safonov, Harnack inequality for elliptic equations and the Hölder property of their solutions, J. Soviet Math. 21 (1983) 851-863.

[26] H. Sharapudinov, The basis property of the Haar system in the space $L^{p(t)}([0,1])$ and the principle of localization in the mean, Mat. Sb. (N.S.) 130 (172) (2) (1986) 275-283.

[27] H. Tian, S. Z. Zheng, Lorentz estimates for the gradient of weak solutions to elliptic obstacle problems with partially BMO coefficients, Boundary Value Problems 2017 (2017) 128. DOI 10.1186/s13661017-0859-9. 
[28] H. Tian, S. Z. Zheng, Uniformly nondegenerate elliptic equations with partially BMO coefficients in nonsmooth domains, Nonlinear Anal. 156 (2017) 90-110.

[29] N. N. Ural'tseva, On impossibility of $W^{2, p}$ estimates for multidimensional elliptic equations with discontinuous coefficients, Zap Nauchn Seminarov LOMI im Steklova, t. 5, ”Nauka," Leningrad (in Russian), 1967.

[30] J. J. Zhang, S. Z. Zheng, Lorentz estimates for fully nonlinear parabolic and elliptic equations, Nonlinear Anal. 148 (2017) 106-125.

[31] J. J. Zhang, S. Z. Zheng, Weighted Lorentz estimates for nondivergence linear elliptic equations with partially BMO coefficients, Commun. Pure Appl. Anal. 16 (3) (2017) 899-914.

[32] C. Zhang, S. L. Zhou, Global weighted estimates for quasilineat elliptic equations with non-standard growth, J. Funct. Anal. 267 (2014) 605-642. 